\title{
Existence Results for Fractional Hahn Difference and Fractional Hahn Integral Boundary Value Problems
}

\author{
Nichaphat Patanarapeelert $^{1}$ and Thanin Sitthiwirattham ${ }^{2}$ \\ ${ }^{1}$ Department of Mathematics, Faculty of Applied Science, King Mongkut's University of Technology North Bangkok, Bangkok, Thailand \\ ${ }^{2}$ Mathematics Department, Faculty of Science and Technology, Suan Dusit University, Bangkok, Thailand \\ Correspondence should be addressed to Thanin Sitthiwirattham; thanin_sit@dusit.ac.th
}

Received 25 September 2017; Revised 15 November 2017; Accepted 27 November 2017; Published 17 December 2017

Academic Editor: Chris Goodrich

Copyright (c) 2017 Nichaphat Patanarapeelert and Thanin Sitthiwirattham. This is an open access article distributed under the Creative Commons Attribution License, which permits unrestricted use, distribution, and reproduction in any medium, provided the original work is properly cited.

\begin{abstract}
The existence and uniqueness results of two fractional Hahn difference boundary value problems are studied. The first problem is a Riemann-Liouville fractional Hahn difference boundary value problem for fractional Hahn integrodifference equations. The second is a fractional Hahn integral boundary value problem for Caputo fractional Hahn difference equations. The Banach fixedpoint theorem and the Schauder fixed-point theorem are used as tools to prove the existence and uniqueness of solution of the problems.
\end{abstract}

\section{Introduction}

The quantum calculus is the subject of calculus without limits and deals with a set of nondifferentiable functions. The quantum operators are widely used in mathematic fields such as hypergeometric series, complex analysis, orthogonal polynomials, combinatorics, hypergeometric functions, and the calculus of variations. The quantum calculus is also found in many applications, such as quantum mechanics and particle physics [1-9].

In 1949, the Hahn difference operator was introduced by Hahn [10]. This operator is a combination of two well-known operators, the forward difference operator and the Jackson $q$ difference operator. The Hahn difference operator is defined by

$$
D_{q, \omega} f(t)=\frac{f(q t+\omega)-f(t)}{t(q-1)+\omega}, \quad t \neq \omega_{0}:=\frac{\omega}{1-q} .
$$

We note that

$$
\begin{aligned}
& D_{q, \omega} f(t)=\Delta_{\omega} f(t) \quad \text { whenever } q=1, \\
& D_{q, \omega} f(t)=D_{q} f(t) \quad \text { whenever } \omega=0, \\
& D_{q, \omega} f(t)=f^{\prime}(t) \quad \text { whenever } q=1, \omega \longrightarrow 0 .
\end{aligned}
$$

The Hahn difference operator has been used in finding families of orthogonal polynomials as well to determine some approximation problems (see [11-13]).

The right inverse of the Hahn difference operator was proposed by Aldwoah in 2009 [14, 15]. This operator is in the terms of the Jackson $q$-integral containing the right inverse of $D_{q}[16]$ and Nörlund sum containing the right inverse of $\Delta_{\omega}$ [16].

In 2010, Malinowska and Torres [17, 18] introduced the Hahn quantum variational calculus. In 2013, Malinowska and Martins [19] presented the generalized transversality conditions for the Hahn quantum variational calculus. Next, Hamza and Ahmed [20, 21] established the theory of linear Hahn difference equations. The authors also study the existence and uniqueness of solution for the initial value problems for Hahn difference equations by employing the method of successive approximations; in addition, they proved Gronwall's and Bernoulli's inequalities with respect to the Hahn difference operator and investigated the mean value theorems for this calculus. In 2016, Hamza and Makharesh [22] investigated Leibniz's rule and Fubini's theorem associated with Hahn difference operator. In the same year, Sitthiwirattham [23] studied the nonlocal boundary value problem for nonlinear Hahn difference equation as given by 


$$
\begin{aligned}
D_{q, \omega}^{2} x(t)+f\left(t, x(t), D_{p, \theta} x(p t+\theta)\right) & =0, \\
t & \in\left[\omega_{0}, T\right]_{q, \omega}, \\
x\left(\omega_{0}\right) & =\varphi(x), \\
x(T) & =\lambda x(\eta), \\
& \eta \in\left(\omega_{0}, T\right)_{q, \omega},
\end{aligned}
$$

where $0<q<1,0<\omega<T, \omega_{0}:=\omega /(1-q), 1 \leq \lambda<$ $\left(T-\omega_{0}\right) /\left(\eta-\omega_{0}\right), p=q^{m}, m \in \mathbb{N}, \theta=\omega((1-p) /(1-q))$, $f:\left[\omega_{0}, T\right]_{q, \omega} \times \mathbb{R} \times \mathbb{R} \rightarrow \mathbb{R}$ is a given function, and $\varphi:$ $C\left(\left[\omega_{0}, T\right]_{q, \omega}, \mathbb{R}\right) \rightarrow \mathbb{R}$ is a given functional.

In 2010, Čermák and Nechvátal [24] established the fractional $(q, h)$-difference operator and the fractional $(q, h)$ integral for $q>1$. In 2011, Čermák et al. [25] studied linear fractional difference equations with discrete Mittag-Leffler functions for $q>1$ in 2011. Rahmat [26, 27] presented the $(q, h)$-Laplace transform and some $(q, h)$-analogues of integral inequalities on discrete time scales for $q>1$. In 2016, Du et al. [28] studied the monotonicity and convexity for nabla fractional $(q, h)$-difference for $q>0, q \neq 1$. Since fractional Hahn operators require the condition $0<q<1$, we note that the operators mentioned above are not fractional Hahn operators. Recently, the fractional Hahn operators have been introduced by Brikshavana and Sitthiwirattham [29].

In order to gain further insight into fractional Hahn operators, in this paper, we study the boundary value problem for fractional Hahn difference equation. Particularly, we consider a Riemann-Liouville fractional Hahn difference boundary value problem for a fractional Hahn integrodifference equation of the form

$$
\begin{aligned}
D_{q, \omega}^{\alpha} u(t) & =F\left(t, u(t), \Psi_{r, \phi}^{\gamma} u(t)\right), \quad t \in\left[\omega_{0}, T\right]_{q, \omega}, \\
u\left(\omega_{0}\right) & =u(T), \\
D_{p, \theta}^{\beta} u\left(\omega_{0}\right) & =D_{p, \theta}^{\beta} u(p T+\theta)
\end{aligned}
$$

and a fractional Hahn integral boundary value problem for a Caputo fractional Hahn difference equation of the form

$$
\begin{aligned}
{ }^{C} D_{q, \omega}^{\alpha} u(t) & =G\left(t, u(t),{ }^{C} D_{r, \phi}^{\gamma} u(r t+\phi)\right), \\
t \in\left[\omega_{0}, T\right]_{q, \omega}, & \\
u\left(\omega_{0}\right) & =\mathscr{A}(u), \\
\mathscr{J}_{p, \theta}^{\beta} u(T) & =\frac{1}{\Gamma_{p}(\beta)} \int_{\omega_{0}}^{T}\left(T-\sigma_{p, \omega}(s)\right) \frac{\beta-1}{p, \theta} u(s) d_{p, \theta} s \\
& =\mathscr{B}(u),
\end{aligned}
$$

where $\left[\omega_{0}, T\right]_{q, \omega}:=\left\{q^{k} T+\omega[k]_{q}: k \in \mathbb{N}_{0}\right\} \cup\left\{\omega_{0}\right\} ; \alpha \in(1,2]$, $\beta, \gamma \in(0,1], \omega>0, p, q, r \in(0,1), p=q^{m}, r=q^{n}, m, n \in$ $\mathbb{N}, \theta=\omega((1-p) /(1-q))$, and $\phi=\omega((1-r) /(1-q)) ;$ $F, G \in C\left(\left[\omega_{0}, T\right]_{q, \omega} \times \mathbb{R} \times \mathbb{R}, \mathbb{R}\right)$ is given function; $\mathscr{A}, \mathscr{B}:$
$C\left(\left[\omega_{0}, T\right]_{q, \omega}, \mathbb{R}\right) \rightarrow \mathbb{R}$ are given functionals; and for $\varphi \in$ $C\left(\left[\omega_{0}, T\right]_{q, \omega} \times\left[\omega_{0}, T\right]_{q, \omega},[0, \infty)\right)$, define

$$
\begin{aligned}
& \Psi_{r, \phi}^{\gamma} u(t):=\left(\mathscr{J}_{r, \phi}^{\gamma} \varphi u\right)(t) \\
& \quad=\frac{1}{\Gamma_{r}(\gamma)} \int_{\omega_{0}}^{t}\left(t-\sigma_{r, \phi}(s)\right)_{r, \phi}^{\frac{\gamma-1}{1}} \varphi(t, s) u(s) d_{r, \phi} s .
\end{aligned}
$$

In Section 2, we provide some basis definitions, properties, and lemma used as material for this work. In Sections 3 and 4 , we prove the existence results of problems (4) and (5), respectively, and we prove the existence and uniqueness of a solution by using the Banach fixed-point theorem and the existence of at least one solution by using the Schauder fixed-point theorem. Finally, some examples are provided to illustrate our results in the last section.

\section{Preliminaries}

In this section, we suggest some notations, definitions, and lemmas which are used in the main results. Let $q \in(0,1)$ and $\omega>0$ and define

$$
\begin{aligned}
{[n]_{q} } & :=\frac{1-q^{n}}{1-q}=q^{n-1}+\cdots+q+1, \\
{[n]_{q} ! } & :=\prod_{k=1}^{n} \frac{1-q^{k}}{1-q},
\end{aligned}
$$

$n \in \mathbb{R}$

The $q$-analogue of the power function $(a-b) \frac{n}{q}$ with $n \in \mathbb{N}_{0}:=$ $[0,1,2, \ldots]$ is defined by

$$
\begin{aligned}
& (a-b) \frac{0}{q}:=1, \\
& (a-b) \frac{n}{q}:=\prod_{k=0}^{n-1}\left(a-b q^{k}\right),
\end{aligned}
$$

$$
a, b \in \mathbb{R}
$$

The $q, \omega$-analogue of the power function $(a-b) \frac{n}{q, \omega}$ with $n \in$ $\mathbb{N}_{0}:=[0,1,2, \ldots]$ is defined by

$$
\begin{aligned}
& (a-b)_{q, \omega}^{\frac{0}{q}}:=1, \\
& (a-b)_{q, \omega}^{\frac{n}{q}}:=\prod_{k=0}^{n-1}\left[a-\left(b q^{k}+\omega[k]_{q}\right)\right],
\end{aligned}
$$

In general, for $\alpha \in \mathbb{R}$, we define

$$
\begin{aligned}
& (a-b) \frac{\alpha}{q}=a^{\alpha} \prod_{n=0}^{\infty} \frac{1-(b / a) q^{n}}{1-(b / a) q^{\alpha+n}}, \quad a \neq 0, \\
& (a-b) \frac{\alpha}{q, \omega}
\end{aligned}
$$




$$
\begin{aligned}
& =\left(a-\omega_{0}\right)^{\alpha} \prod_{n=0}^{\infty} \frac{1-\left(\left(b-\omega_{0}\right) /\left(a-\omega_{0}\right)\right) q^{n}}{1-\left(\left(b-\omega_{0}\right) /\left(a-\omega_{0}\right)\right) q^{\alpha+n}} \\
& =\left(\left(a-\omega_{0}\right)-\left(b-\omega_{0}\right)\right)_{q}^{\alpha}, \quad a \neq \omega_{0} .
\end{aligned}
$$

We note that $a_{q} \frac{\alpha}{q}=a^{\alpha}$ and $\left(a-\omega_{0}\right) \frac{\alpha}{q, \omega}=\left(a-\omega_{0}\right)^{\alpha}$ and use the notation $(0) \frac{\alpha}{q}=\left(\omega_{0}\right) \frac{\alpha}{q}, \omega=0$ for $\alpha>0$. The $q$-gamma and $q$-beta functions are defined by

$$
\begin{aligned}
\Gamma_{q}(x) & :=\frac{(1-q) \frac{x-1}{q}}{(1-q)^{x-1}}, \quad x \in \mathbb{R} \backslash\{0,-1,-2, \ldots\}, \\
B_{q}(x, s) & :=\int_{0}^{1} t^{x-1}(1-q t) \frac{s-1}{q} d_{q} t=\frac{\Gamma_{q}(x) \Gamma_{q}(s)}{\Gamma_{q}(x+s)} .
\end{aligned}
$$

Definition 1. For $q \in(0,1), \omega>0$, and $f$ defined on an interval $I \subseteq \mathbb{R}$ containing $\omega_{0}:=\omega /(1-q)$, the Hahn difference of $f$ is defined by

$$
D_{q, \omega} f(t)=\frac{f(q t+\omega)-f(t)}{t(q-1)+\omega} \text { for } t \neq \omega_{0},
$$

and $D_{q, \omega} f\left(\omega_{0}\right)=f^{\prime}\left(\omega_{0}\right)$. Providing that $f$ is differentiable at $\omega_{0}$, we call $D_{q, \omega} f$ the $q, \omega$-derivative of $f$ and say that $f$ is $q, \omega$-differentiable on $I$.

Remarks 2. We give some properties for the Hahn difference as follows:

(1) $D_{q, \omega}[f(t)+g(t)]=D_{q, \omega} f(t)+D_{q, \omega} g(t)$.

(2) $D_{q, \omega}[\alpha f(t)]=\alpha D_{q, \omega} f(t)$.

(3) $D_{q, \omega}[f(t) g(t)]=f(t) D_{q, \omega} g(t)+g(q t+\omega) D_{q, \omega} f(t)$.

(4) $D_{q, \omega}[f(t) / g(t)]=\left(g(t) D_{q, \omega} f(t)-f(t) D_{q, \omega} g(t)\right) /$ $g(t) g(q t+\omega)$.

Letting $a, b \in I \subseteq \mathbb{R}$ with $a<\omega_{0}<b$ and $[k]_{q}=(1-$ $\left.q^{k}\right) /(1-q), k \in \mathbb{N}_{0}:=\mathbb{N} \cup\{0\}$, we define the $q$, $\omega$-interval by

$$
\begin{aligned}
I_{q, \omega}^{a, b}= & {[a, b]_{q, \omega} } \\
:= & \left\{q^{k} a+\omega[k]_{q}: k \in \mathbb{N}_{0}\right\} \\
& \cup\left\{q^{k} b+\omega[k]_{q}: k \in \mathbb{N}_{0}\right\} \cup\left\{\omega_{0}\right\} \\
= & {\left[a, \omega_{0}\right]_{q, \omega} \cup\left[\omega_{0}, b\right]_{q, \omega}=(a, b)_{q, \omega} \cup\{a, b\} } \\
= & {[a, b)_{q, \omega} \cup\{b\}=(a, b]_{q, \omega} \cup\{a\}, } \\
I_{q, \omega}^{T}:= & I_{q, \omega}^{\omega_{0}, T}=\left[\omega_{0}, T\right]_{q, \omega} .
\end{aligned}
$$

Observe that, for each $s \in[a, b]_{q, \omega}$, the sequence $\left\{\sigma_{q, \omega}^{k}(s)\right\}_{k=0}^{\infty}=\left\{q^{k} s+\omega[k]_{q}\right\}_{k=0}^{\infty}$ is uniformly convergent to $\omega_{0}$.

We also define the forward jump operator as $\sigma_{q, \omega}^{k}(t):=$ $q^{k} t+\omega[k]_{q}$ and the backward jump operator as $\rho_{q, \omega}^{k}(t):=(t-$ $\left.\omega[k]_{q}\right) / q^{k}$ for $k \in \mathbb{N}$.
Definition 3. Let $I$ be any closed interval of $\mathbb{R}$ containing $a, b$, and $\omega_{0}$. Assuming that $f: I \rightarrow \mathbb{R}$ is a given function, we define $q, \omega$-integral of $f$ from $a$ to $b$ by

$$
\int_{a}^{b} f(t) d_{q, \omega} t:=\int_{\omega_{0}}^{b} f(t) d_{q, \omega} t-\int_{\omega_{0}}^{a} f(t) d_{q, \omega} t
$$

where

$$
\begin{aligned}
& \int_{\omega_{0}}^{x} f(t) d_{q, \omega} t \\
& \quad:=[x(1-q)-\omega] \sum_{k=0}^{\infty} q^{k} f\left(x q^{k}+\omega[k]_{q}\right), \quad x \in I .
\end{aligned}
$$

Providing that the series converges at $x=a$ and $x=b$, we consider $f$ as $q, \omega$-integrable on $[a, b]$ and the sum to the right-hand side of above equation will be called the JacksonNörlund sum.

We note that the actual domain of function $f$ defined on $[a, b]_{q, \omega} \subset I$.

We next introduce the fundamental theorem of Hahn calculus in the following lemma.

Lemma 4 (see [14]). Let $f: I \rightarrow \mathbb{R}$ be continuous at $\omega_{0}$. Define

$$
F(x):=\int_{\omega_{0}}^{x} f(t) d_{q, \omega} t, \quad x \in I
$$

Then, $F$ is continuous at $\omega_{0}$. Furthermore, $D_{q, \omega} F(x)$ exists for every $x \in I$ and

$$
D_{q, \omega} F(x)=f(x) .
$$

Conversely, one has

$$
\int_{a}^{b} D_{q, \omega} F(t) d_{q, \omega} t=F(b)-F(a) \quad \forall a, b \in I .
$$

Lemma 5 (see [23]). Let $q \in(0,1), \omega>0$, and $f: I \rightarrow \mathbb{R}$ be continuous at $\omega_{0}$. Then,

$$
\int_{\omega_{0}}^{t} \int_{\omega_{0}}^{r} f(s) d_{q, \omega} s d_{q, \omega} r=\int_{\omega_{0}}^{t} \int_{q s+\omega}^{t} f(s) d_{q, \omega} r d_{q, \omega} s .
$$

Lemma 6 (see [23]). Let $q \in(0,1)$ and $\omega>0$. Then,

$$
\begin{gathered}
\int_{\omega_{0}}^{t} d_{q, \omega} s=t-\omega_{0} \\
\int_{\omega_{0}}^{t}\left[t-\sigma_{q, \omega}(s)\right] d_{q, \omega} s=\frac{\left(t-\omega_{0}\right)^{2}}{1+q} .
\end{gathered}
$$

We next introduce fractional Hahn integral and fractional Hahn difference of Riemann-Liouville and Caputo types as follows. 
Definition 7. For $\alpha, \omega>0, q \in(0,1)$, and $f$ defined on $\left[\omega_{0}, T\right]_{q, \omega}$, the fractional Hahn integral is defined by

$$
\begin{aligned}
& \mathcal{F}_{q, \omega}^{\alpha} f(t):=\frac{1}{\Gamma_{q}(\alpha)} \int_{\omega_{0}}^{t}\left(t-\sigma_{q, \omega}(s)\right)_{q, \omega}^{\alpha-1} f(s) d_{q, \omega} s \\
& =\frac{[t(1-q)-\omega]}{\Gamma_{q}(\alpha)} \sum_{n=0}^{\infty} q^{n}\left(t-\sigma_{q, \omega}^{n+1}(t)\right)_{q, \omega}^{\alpha-1} f\left(\sigma_{q, \omega}^{n}(t)\right),
\end{aligned}
$$

and $\left(\mathscr{I}_{q, \omega}^{0} f\right)(t)=f(t)$.

Definition 8. For $\alpha, \omega>0, q \in(0,1), N-1<\alpha<N, N \in \mathbb{N}$, and $f$ defined on $\left[\omega_{0}, T\right]_{q, \omega}$, the fractional Hahn difference of the Riemann-Liouville type of order $\alpha$ is defined by

$$
\begin{aligned}
D_{q, \omega}^{\alpha} f(t) & :=\left(D_{q, \omega}^{N} \mathcal{J}_{q, \omega}^{N-\alpha} f\right)(t) \\
& =\frac{1}{\Gamma_{q}(-\alpha)} \int_{\omega_{0}}^{t}\left(t-\sigma_{q, \omega}(s)\right)_{q, \omega}^{-\alpha-1} f(s) d_{q, \omega} s .
\end{aligned}
$$

The fractional Hahn difference of the Caputo type of order $\alpha$ is defined by

$$
\begin{aligned}
& { }^{C} D_{q, \omega}^{\alpha} f(t):=\left(\mathcal{F}_{q, \omega}^{N-\alpha} D_{q, \omega}^{N} f\right)(t) \\
& =\frac{1}{\Gamma_{q}(N-\alpha)} \int_{\omega_{0}}^{t}\left(t-\sigma_{q, \omega}(s)\right)_{q, \omega}^{N-\alpha-1} D_{q, \omega}^{N} f(s) d_{q, \omega} s,
\end{aligned}
$$

and $D_{q, \omega}^{0} f(t)={ }^{C} D_{q, \omega}^{0} f(t)=f(t)$.

Lemma 9 (see [29]). Let $\alpha>0, q \in(0,1), \omega>0$, and $f$ : $I_{q, \omega}^{T} \rightarrow \mathbb{R}$. Then

$$
\begin{aligned}
\mathscr{I}_{q, \omega}^{\alpha} D_{q, \omega}^{\alpha} f(t)= & f(t)+C_{1}\left(t-\omega_{0}\right)^{\alpha-1}+\cdots \\
& +C_{N}\left(t-\omega_{0}\right)^{\alpha-N},
\end{aligned}
$$

for some $C_{i} \in \mathbb{R}, i=\mathbb{N}_{1, N}$, and $N-1<\alpha \leq N, N \in \mathbb{N}$.

Lemma 10 (see [29]). Let $\alpha>0, q \in(0,1), \omega>0$, and $f$ : $I_{q, \omega}^{T} \rightarrow \mathbb{R}$. Then

$$
\begin{aligned}
\mathscr{F}_{q, \omega}^{\alpha}{ }^{C} D_{q, \omega}^{\alpha} f(t)= & f(t)+C_{0}+C_{1}\left(t-\omega_{0}\right)+\cdots \\
& +C_{N-1}\left(t-\omega_{0}\right)^{N-1}
\end{aligned}
$$

for some $C_{i} \in \mathbb{R}, i=\mathbb{N}_{0, N-1}$, and $N-1<\alpha \leq N, N \in \mathbb{N}$.

Next, we give some auxiliary lemmas for simplifying our calculations.

Lemma 11 (see [29]). Let $\alpha, \beta>0, p, q \in(0,1)$, and $\omega>0$. Then,

$$
\begin{gathered}
\int_{\omega_{0}}^{t}\left(t-\sigma_{q, \omega}(s)\right)_{q, \omega}^{\frac{\alpha-1}{}}\left(s-\omega_{0}\right) \frac{\beta}{q, \omega} d_{q, \omega} s \\
=\left(t-\omega_{0}\right)^{\alpha+\beta} B_{q}(\beta+1, \alpha),
\end{gathered}
$$

$$
\begin{aligned}
& \int_{\omega_{0}}^{t} \int_{\omega_{0}}^{x}\left(t-\sigma_{p, \omega}(x)\right)_{p, \omega}^{\frac{\alpha-1}{p}}\left(x-\sigma_{q, \omega}(s) \frac{\beta-1}{q, \omega} d_{q, \omega} s d_{p, \omega} x\right. \\
& =\frac{\left(t-\omega_{0}\right)^{\alpha+\beta}}{[\beta]_{q}} B_{p}(\beta+1, \alpha) .
\end{aligned}
$$

In the following, we give a lemma that deals with the linear variant of problem (4) and gives a representation of the solution.

Lemma 12. Let $\Omega \neq 0, \alpha \in(1,2], \beta \in(0,1], \omega>0, p, q \in$ $(0,1), p=q^{m}, m \in \mathbb{N}$, and $\theta=\omega((1-p) /(1-q))$ and let $h \in C\left(\left[\omega_{0}, T\right]_{q, \omega}, \mathbb{R}\right)$ be a given function. Then the problem

$$
\begin{aligned}
D_{q, \omega}^{\alpha} u(t) & =h(t), \quad t \in\left[\omega_{0}, T\right]_{q, \omega}, \\
u\left(\omega_{0}\right) & =u(T), \\
D_{p, \theta}^{\beta} u\left(\omega_{0}\right) & =D_{p, \theta}^{\beta} u(p T+\theta)
\end{aligned}
$$

has the unique solution

$$
\begin{aligned}
u(t)= & \frac{\left(t-\omega_{0}\right)^{\alpha-1}}{\Omega}\left[\left(T-\omega_{0}\right)^{\alpha-2} \mathscr{P}[h]-\Lambda_{2} \mathscr{Q}[h]\right] \\
& -\frac{\left(t-\omega_{0}\right)^{\alpha-2}}{\Omega}\left[\left(T-\omega_{0}\right)^{\alpha-1} \mathscr{P}[h]-\Lambda_{1} \mathcal{Q}[h]\right] \\
& +\frac{1}{\Gamma_{q}(\alpha)} \int_{\omega_{0}}^{t}\left(t-\sigma_{q, \omega}(s)\right)_{q, \omega}^{\frac{\alpha-1}{h}} h(s) d_{q, \omega} s
\end{aligned}
$$

where the functions $\mathscr{P}[h]$ and $Q[h]$ are defined by

$$
\begin{gathered}
\mathscr{P}[h]:=\frac{1}{\Gamma_{q}(\alpha) \Gamma_{p}(-\beta)} \\
\cdot \int_{\omega_{0}}^{p T+\theta} \int_{\omega_{0}}^{x}\left(p T+\theta-\sigma_{p, \theta}(x)\right)_{p, \theta}^{\frac{-\beta-1}{p}} \\
\cdot\left(x-\sigma_{q, \omega}(s)\right)_{q, \omega}^{\frac{\alpha-1}{}} h(s) d_{p, \theta} s d_{q, \omega} x, \\
\mathbb{Q}[h]:=\frac{1}{\Gamma_{q}(\alpha)} \int_{\omega_{0}}^{T}\left(T-\sigma_{q, \omega}(s)\right)_{q, \omega}^{\frac{\alpha-1}{2}} h(s) d_{q, \omega} s,
\end{gathered}
$$

respectively. The constants $\Omega, \Lambda_{1}$, and $\Lambda_{2}$ are defined by

$$
\begin{aligned}
\Omega & :=\left(T-\omega_{0}\right)^{\alpha-1} \Lambda_{2}-\left(T-\omega_{0}\right)^{\alpha-2} \Lambda_{1}, \\
\Lambda_{1} & :=\frac{1}{\Gamma_{p}(-\beta)} \\
& \cdot \int_{\omega_{0}}^{p T+\theta}\left(p T+\theta-\sigma_{p, \theta}(s)\right) \frac{-\beta-1}{p, \theta}\left(s-\omega_{0}\right)^{\alpha-1} d_{p, \theta} s \\
& =\frac{\Gamma_{p}(\alpha)\left(p\left(T-\omega_{0}\right)\right)^{\alpha-\beta-1}}{\Gamma_{p}(\alpha-\beta)},
\end{aligned}
$$




$$
\begin{aligned}
\Lambda_{2} & :=\frac{1}{\Gamma_{p}(-\beta)} \\
& \cdot \int_{\omega_{0}}^{p T+\theta}\left(p T+\theta-\sigma_{p, \theta}(s)\right) \frac{-\beta-1}{p, \theta}\left(s-\omega_{0}\right)^{\alpha-2} d_{p, \theta} s \\
& =\frac{\Gamma_{p}(\alpha-1)\left(p\left(T-\omega_{0}\right)\right)^{\alpha-\beta-2}}{\Gamma_{p}(\alpha-\beta-1)},
\end{aligned}
$$

respectively.

Proof. Taking fractional Hahn $q$, $\omega$-integral of order $\alpha$ for (27), we obtain

$$
\begin{aligned}
u(t)= & \mathscr{I}_{q, \omega}^{\alpha} h(t)+C_{1}\left(t-\omega_{0}\right)^{\alpha-1}+C_{2}\left(t-\omega_{0}\right)^{\alpha-2} \\
= & \frac{1}{\Gamma_{q}(\alpha)} \int_{\omega_{0}}^{t}\left(t-\sigma_{q, \omega}(s)\right)_{q, \omega}^{\frac{\alpha-1}{2}} h(s) d_{q, \omega} s \\
& +C_{1}\left(t-\omega_{0}\right)^{\alpha-1}+C_{2}\left(t-\omega_{0}\right)^{\alpha-2} .
\end{aligned}
$$

Taking fractional Hahn $p, \theta$-difference of $\operatorname{order} \beta$ for (34), we obtain

$$
\begin{aligned}
& D_{p, \theta}^{\beta} u(t)=\frac{1}{\Gamma_{q}(\alpha) \Gamma_{p}(-\beta)} \int_{\omega_{0}}^{t} \int_{\omega_{0}}^{x}\left(t-\sigma_{p, \theta}(x)\right) \frac{-\beta-1}{p, \theta} \\
& \cdot\left(x-\sigma_{q, \omega}(s)\right)_{q, \omega}^{\frac{\alpha-1}{d}} h(s) d_{q, \omega} s d_{p, \theta} x \\
& +\frac{C_{1}}{\Gamma_{q}(-\beta)} \int_{\omega_{0}}^{t}\left(t-\sigma_{p, \theta}(s)\right) \frac{-\beta-1}{p, \theta}\left(s-\omega_{0}\right)^{\alpha-1} d_{p, \theta} s \\
& +\frac{C_{2}}{\Gamma_{q}(-\beta)} \int_{\omega_{0}}^{t}\left(t-\sigma_{p, \theta}(s)\right) \frac{-\beta-1}{p, \theta}\left(s-\omega_{0}\right)^{\alpha-2} d_{p, \theta} s .
\end{aligned}
$$

Substituting $t=\omega_{0}, T$ into (34) and using the first condition of (27), we have

$$
\begin{aligned}
C_{1} & \left(T-\omega_{0}\right)^{\alpha-1}+C_{2}\left(T-\omega_{0}\right)^{\alpha-2} \\
& =-\frac{1}{\Gamma_{q}(\alpha)} \int_{\omega_{0}}^{T}\left(T-\sigma_{q, \omega}(s)\right)_{q, \omega}^{\frac{\alpha-1}{}} h(s) d_{q, \omega} s .
\end{aligned}
$$

By letting $t=\omega_{0}, p T+\theta$ into (35) and employing the second condition of (27), we obtain

$$
\begin{aligned}
& \frac{C_{1}}{\Gamma_{p}(-\beta)} \int_{\omega_{0}}^{p T+\theta}\left(p T+\theta-\sigma_{p, \theta}(s)\right) \frac{-\beta-1}{p, \theta}(s \\
& \left.-\omega_{0}\right)^{\alpha-1} d_{p, \theta} s+\frac{C_{2}}{\Gamma_{p}(-\beta)} \int_{\omega_{0}}^{p T+\theta}(p T+\theta \\
& \left.-\sigma_{p, \theta}(s)\right) \frac{-\beta-1}{p, \theta}\left(s-\omega_{0}\right)^{\alpha-2} d_{p, \theta} s \\
& =-\frac{1}{\Gamma_{q}(\alpha) \Gamma_{p}(-\beta)}
\end{aligned}
$$

$$
\begin{aligned}
& \cdot \int_{\omega_{0}}^{p T+\theta} \int_{\omega_{0}}^{x}\left(p T+\theta-\sigma_{p, \theta}(x)\right) \frac{-\beta-1}{p, \theta} \\
& \cdot\left(x-\sigma_{q, \omega}(s)\right)_{q, \omega}^{\frac{\alpha-1}{2}} h(s) d_{q, \omega} s d_{p, \theta} x .
\end{aligned}
$$

To find $C_{1}$ and $C_{2}$, we solve the system of equations (36) and (37). Then, we obtain

$$
\begin{aligned}
& C_{1}=\frac{1}{\Omega}\left[\left(T-\omega_{0}\right)^{\alpha-2} \mathscr{P}[h]-\Lambda_{2} \mathscr{Q}[h]\right], \\
& C_{2}=-\frac{1}{\Omega}\left[\left(T-\omega_{0}\right)^{\alpha-1} \mathscr{P}[h]-\Lambda_{1} \mathscr{Q}[h]\right],
\end{aligned}
$$

where $\mathscr{P}[h], \mathcal{Q}[h], \Omega, \Lambda_{1}$, and $\Lambda_{2}$ are defined as (29)-(33), respectively.

Substituting the constants $C_{1}$ and $C_{2}$ into (34) and by Cramer's rule, it is implied that (28) is the uniqueness solution.

We next prove a lemma that deals with the linear variant of problem (5) and gives a representation of the solution.

Lemma 13. Let $\alpha \in(1,2], \beta \in(0,1], \omega>0, p, q \in$ $(0,1), p=q^{m}, m \in \mathbb{N}$, and $\theta=\omega((1-p) /(1-q))$ and let $h \in C\left(\left[\omega_{0}, T\right]_{q, \omega}, \mathbb{R}\right)$ be a given function and $\mathscr{A}, \mathscr{B}$ : $C\left(\left[\omega_{0}, T\right]_{q, \omega}, \mathbb{R}\right) \rightarrow \mathbb{R}$ be given functionals. Then, the problem

$$
\begin{aligned}
{ }^{C} D_{q, \omega}^{\alpha} u(t) & =h(t), \quad t \in\left[\omega_{0}, T\right]_{q, \omega}, \\
u\left(\omega_{0}\right) & =\mathscr{A}(u), \\
\mathscr{J}_{p, \theta}^{\beta} u(T) & =\frac{1}{\Gamma_{p}(\beta)} \int_{\omega_{0}}^{T}\left(T-\sigma_{p, \theta}(s)\right) \frac{\beta-1}{p, \theta} u(s) d_{p, \theta} s \\
& =\mathscr{B}(u)
\end{aligned}
$$

has the unique solution

$$
\begin{aligned}
& u(t)=\mathscr{A}(u)+\frac{\left(t-\omega_{0}\right) \Gamma_{q}(\beta+2)}{\left(T-\omega_{0}\right)^{\beta+1}}\{\mathscr{B}(u) \\
& -\frac{\left(T-\omega_{0}\right)^{\beta}}{\Gamma_{q}(\beta+1)} \mathscr{A}(u)-\frac{1}{\Gamma_{q}(\alpha) \Gamma_{p}(\beta)} \\
& \times \int_{\omega_{0}}^{T} \int_{\omega_{0}}^{x}\left(T-\sigma_{p, \theta}(x)\right)_{p, \theta}^{\beta-1}\left(x-\sigma_{q, \omega}(s)\right)_{q, \omega}^{\frac{\alpha-1}{}} \\
& \left.\cdot h(s) d_{q, \omega} s d_{p, \theta} x\right\}+\frac{1}{\Gamma_{q}(\alpha)} \int_{\omega_{0}}^{t}(t \\
& \left.-\sigma_{q, \omega}(s)\right)_{q, \omega}^{\frac{\alpha-1}{}} h(s) d_{q, \omega} s .
\end{aligned}
$$

Proof. Taking fractional Hahn $q$, $\omega$-integral of order $\alpha$ for (39), we obtain

$$
u(t)=\mathscr{I}_{q, \omega}^{\alpha} h(t)+C_{1}\left(t-\omega_{0}\right)+C_{2} .
$$


Taking fractional Hahn $p, \theta$-integral of order $\beta$ for (41), we have

$$
\begin{gathered}
\mathscr{J}_{p, \theta}^{\beta} u(t)=\mathscr{J}_{p, \theta}^{\beta} \mathscr{J}_{q, \omega}^{\alpha} h(t)+\frac{1}{\Gamma_{q}(\beta)} \int_{\omega_{0}}^{t}(t \\
\left.-\sigma_{p, \omega}(s)\right)_{p, \omega}^{\beta-1}\left[C_{1}\left(s-\omega_{0}\right)+C_{2}\right] d_{p, \omega} s \\
=\frac{1}{\Gamma_{q}(\alpha) \Gamma_{p}(\beta)} \int_{\omega_{0}}^{t} \int_{\omega_{0}}^{x}\left(t-\sigma_{p, \theta}(x)\right) \frac{\beta-1}{p, \theta} \\
\cdot\left(x-\sigma_{q, \omega}(s)\right)_{q, \omega}^{\frac{\alpha-1}{\omega}} h(s) d_{q, \omega} s d_{p, \theta} x \\
+\frac{\left(t-\omega_{0}\right)^{\beta+1}}{\Gamma_{p}(\beta+2)} C_{1}+\frac{\left(t-\omega_{0}\right)^{\beta}}{\Gamma_{p}(\beta+1)} C_{2} .
\end{gathered}
$$

Letting $t=\omega_{0}$ into (41) and employing the first condition of (39), we get

$$
C_{2}=\mathscr{A}(u)
$$

Substituting $t=T$ into (42) and employing the second condition of (39), we obtain

$$
\begin{aligned}
C_{1} & =\frac{\Gamma_{q}(\beta+2)}{\left(T-\omega_{0}\right)^{\beta+1}}\left\{\mathscr{B}(u)-\frac{\left(T-\omega_{0}\right)^{\beta}}{\Gamma_{q}(\beta+1)} \mathscr{A}(u)\right. \\
& -\frac{1}{\Gamma_{q}(\alpha) \Gamma_{p}(\beta)} \times \int_{\omega_{0}}^{T} \int_{\omega_{0}}^{x}\left(T-\sigma_{p, \theta}(x)\right) \frac{\beta-1}{p, \theta} \\
& \left.\cdot\left(x-\sigma_{q, \omega}(s)\right)_{q, \omega}^{\frac{\alpha-1}{q}} h(s) d_{q, \omega} s d_{p, \theta} x\right\} .
\end{aligned}
$$

Substituting the constants $C_{1}$ and $C_{2}$ into (41) and by Cramer's rule, it is implied that (40) is the uniqueness solution.

We next introduce Schauder's fixed-point theorem used to prove the existence of a solution to (4) and (5).

Lemma 14 ([30] (Arzelá-Ascoli theorem)). A set of functions in $C[a, b]$ with the sup norm is relatively compact if and only if it is uniformly bounded and equicontinuous on $[a, b]$.

Lemma 15 (see [30]). If a set is closed and relatively compact, then it is compact.

Lemma 16 ([31] (Schauder's fixed-point theorem)). Let $(D, d)$ be a complete metric space, let $U$ be a closed convex subset of $D$, and let $T: D \rightarrow D$ be the map such that the set $T u: u \in U$ is relatively compact in $D$. Then the operator $T$ has at least one fixed point $u^{*} \in U: T u^{*}=u^{*}$.

\section{Existence Results for Problem (4)}

In this section, we prove the existence results for problem (4). Let $\mathscr{X}=C\left(I_{q, \omega}^{T}, \mathbb{R}\right)$ be a Banach space of all function $u$ with the norm defined by

$$
\|u\|_{\mathscr{X}}=\max _{t \in I_{q, \omega}^{T}}\{|u(t)|\}
$$

where $\omega>0, p, q, r \in(0,1), p=q^{m}, r=q^{n}, m, n \in \mathbb{N}$, $\theta=\omega((1-p) /(1-q))$, and $\phi=\omega((1-r) /(1-q))$. Define an operator $\mathscr{F}: \mathscr{X} \rightarrow \mathscr{X}$ by

$$
\begin{aligned}
& (\mathscr{F} u)(t):=\frac{\left(t-\omega_{0}\right)^{\alpha-1}}{\Omega}\left[\left(T-\omega_{0}\right)^{\alpha-2} \mathscr{P}^{*}\left[F_{u}\right]\right. \\
& \left.-\Lambda_{2} \mathscr{Q}^{*}\left[F_{u}\right]\right]-\frac{\left(t-\omega_{0}\right)^{\alpha-2}}{\Omega}\left[\left(T-\omega_{0}\right)^{\alpha-1} \mathscr{P}^{*}\left[F_{u}\right]\right. \\
& \left.-\Lambda_{1} Q^{*}\left[F_{u}\right]\right]+\frac{1}{\Gamma_{q}(\alpha)} \int_{\omega_{0}}^{t}\left(t-\sigma_{q, \omega}(s)\right)_{q, \omega}^{\frac{\alpha-1}{\omega}} \\
& \cdot F\left(s, u(s), \Psi_{r, \phi}^{\gamma} u(s)\right) d_{q, \omega} s,
\end{aligned}
$$

where $\Omega, \Lambda_{1}$, and $\Lambda_{2}$ are defined as (31)-(33), respectively, and the functions $\mathscr{P}^{*}\left[F_{u}\right]$ and $Q^{*}\left[F_{u}\right]$ are defined by

$$
\begin{aligned}
& \mathscr{P}^{*}\left[F_{u}\right]:=\frac{1}{\Gamma_{q}(\alpha) \Gamma_{p}(-\beta)} \\
& \cdot \int_{\omega_{0}}^{p T+\theta} \int_{\omega_{0}}^{x}\left(p T+\theta-\sigma_{p, \theta}(x)\right) \frac{-\beta-1}{p, \theta} \\
& \cdot\left(x-\sigma_{q, \omega}(s)\right)_{q, \omega}^{\frac{\alpha-1}{2}} \times F\left(s, u(s), \Psi_{r, \phi}^{\gamma} u(s)\right) d_{q, \omega} x d_{p, \theta} s, \\
& \left.{Q^{*}}^{*} F_{u}\right]:=\frac{1}{\Gamma_{q}(\alpha)} \int_{\omega_{0}}^{T}\left(T-\sigma_{q, \omega}(s)\right)_{q, \omega}^{\frac{\alpha-1}{\omega}} \\
& \cdot F\left(s, u(s), \Psi_{r, \phi}^{\gamma} u(s)\right) d_{q, \omega} s .
\end{aligned}
$$

Obviously, problem (4) has solutions if and only if the operator $\mathscr{F}$ has fixed points.

Theorem 17. Assume that $F: I_{q, \omega}^{T} \times \mathbb{R} \times \mathbb{R} \rightarrow \mathbb{R}$ is continuous and $\varphi: I_{q, \omega}^{T} \times I_{q, \omega}^{T} \rightarrow[0, \infty)$ is continuous with $\varphi_{0}=$ $\max \left\{\varphi(t, s):(t, s) \in I_{q, \omega}^{T} \times I_{q, \omega}^{T}\right\}$. In addition, suppose that the following conditions hold:

$\left(H_{1}\right)$ There exist constants $\lambda_{1}, \lambda_{2}>0$ such that, for each $t \in I_{q, \omega}^{T}$ and $u, v \in \mathbb{R}$,

$$
\begin{aligned}
& \left|F\left(t, u, \Psi_{r, \phi}^{\gamma} u\right)-F\left(t, v, \Psi_{r, \phi}^{\gamma} v\right)\right| \\
& \quad \leq \lambda_{1}|u-v|+\lambda_{2}\left|\Psi_{r, \phi}^{\gamma} u-\Psi_{r, \phi}^{\gamma} v\right| .
\end{aligned}
$$

$\left(H_{2}\right) \mathscr{L} \Theta<1$, where

$$
\mathscr{L}:=\lambda_{1}+\lambda_{2} \varphi_{0} \frac{\left(T-\omega_{0}\right)^{\gamma}}{\Gamma_{r}(\gamma+1)},
$$




$$
\begin{aligned}
\Theta & :=\frac{\left(T-\omega_{0}\right)^{2 \alpha-1}}{|\Omega|} \mid \frac{p^{\alpha+\beta} \Gamma_{q}(-\beta)\left(T-\omega_{0}\right)^{\alpha-\beta-2}}{\Gamma_{p}(-\beta) \Gamma_{q}(\alpha-\beta+1)} \\
& -\frac{\Lambda_{2}}{\Gamma_{q}(\alpha+1)} \mid \\
& +\frac{\left(T-\omega_{0}\right)^{2 \alpha-2}}{|\Omega|} \mid \frac{p^{\alpha+\beta} \Gamma_{q}(-\beta)\left(T-\omega_{0}\right)^{\alpha-\beta-1}}{\Gamma_{p}(-\beta) \Gamma_{q}(\alpha-\beta+1)} \\
& -\frac{\Lambda_{1}}{\Gamma_{q}(\alpha+1)} \mid+\frac{\left(T-\omega_{0}\right)^{\alpha}}{\Gamma_{q}(\alpha+1)} .
\end{aligned}
$$

Then, problem (4) has a unique solution.

Proof. To prove that $F$ is contraction, we consider

$$
\begin{aligned}
\mathscr{H} & |u-v|(t) \\
& :=\left|F\left(t, u(t), \Psi_{r, \phi}^{\gamma} u(t)\right)-F\left(t, v(t), \Psi_{r, \phi}^{\gamma} v(t)\right)\right| \\
& \leq\left\|F\left(t, u, \Psi_{r, \phi}^{\gamma} u\right)-F\left(t, v, \Psi_{r, \phi}^{\gamma} v\right)\right\|=: \mathscr{H}\|u-v\|,
\end{aligned}
$$

for each $t \in I_{q, \omega}^{T}$ and $u, v \in \mathscr{X}$. We find that

$$
\begin{aligned}
& \left|\mathscr{P}^{*}\left[F_{u}\right]-\mathscr{P}^{*}\left[F_{v}\right]\right|=\frac{1}{\Gamma_{q}(\alpha) \Gamma_{p}(-\beta)} \\
& \cdot \int_{\omega_{0}}^{p T+\theta} \int_{\omega_{0}}^{x}\left(p T+\theta-\sigma_{p, \theta}(x)\right)_{p, \theta}^{-\beta-1} \\
& \times\left(x-\sigma_{q, \omega}(s)\right)_{q, \omega}^{\frac{\alpha-1}{H}} \mathscr{H}|u-v|(s) d_{q, \omega} s d_{p, \theta} x \\
& \leq\left(\lambda_{1}|u-v|+\lambda_{2}\left|\Psi_{r, \phi}^{\gamma} u-\Psi_{r, \phi}^{\gamma} v\right|\right) \\
& \cdot \frac{\Gamma_{q}(-\beta)\left(p\left(T-\omega_{0}\right)\right)^{\alpha-\beta}}{\Gamma_{p}(-\beta) \Gamma_{q}(\alpha-\beta+1)}, \\
& \left|Q^{*}\left[F_{u}\right]-\mathscr{Q}^{*}\left[F_{v}\right]\right|=\frac{1}{\Gamma_{q}(\alpha)} \\
& \cdot \int_{\omega_{0}}^{T}\left(T-\sigma_{q, \omega}(s)\right)_{q, \omega}^{\frac{\alpha-1}{H}|u-v|(s) d_{q, \omega} s} \\
& \leq\left(\lambda_{1}|u-v|+\lambda_{2}\left|\Psi_{r, \phi}^{\gamma} u-\Psi_{r, \phi}^{\gamma} v\right|\right) \frac{\left(T-\omega_{0}\right)^{\alpha}}{\Gamma_{q}(\alpha+1)} .
\end{aligned}
$$

Finally, for each $t \in I_{q, \omega}^{T}$ and $u, v \in \mathscr{X}$,

$$
\begin{aligned}
& |(\mathscr{F} u)(t)-(\mathscr{F} v)(t)| \leq\left(\lambda_{1}|u-v|\right. \\
& \left.\quad+\lambda_{2}\left|\Psi_{r, \phi}^{\gamma} u-\Psi_{r, \phi}^{\gamma} v\right|\right)\left\{\frac{\left(t-\omega_{0}\right)^{\alpha-1}\left(T-\omega_{0}\right)^{\alpha}}{|\Omega|}\right.
\end{aligned}
$$

$$
\begin{aligned}
& \times\left|\frac{p^{\alpha+\beta} \Gamma_{q}(-\beta)\left(T-\omega_{0}\right)^{\alpha-\beta-2}}{\Gamma_{p}(-\beta) \Gamma_{q}(\alpha-\beta+1)}-\frac{\Lambda_{2}}{\Gamma_{q}(\alpha+1)}\right| \\
& +\frac{\left(t-\omega_{0}\right)^{\alpha-2}\left(T-\omega_{0}\right)^{\alpha}}{\Omega} \\
& \times\left|\frac{p^{\alpha+\beta} \Gamma_{q}(-\beta)\left(T-\omega_{0}\right)^{\alpha-\beta-1}}{\Gamma_{p}(-\beta) \Gamma_{q}(\alpha-\beta+1)}-\frac{\Lambda_{1}}{\Gamma_{q}(\alpha+1)}\right| \\
& \left.+\frac{\left(t-\omega_{0}\right)^{\alpha}}{\Gamma_{q}(\alpha+1)}\right\} \leq\left(\lambda_{1}+\lambda_{2} \varphi_{0} \frac{\left(T-\omega_{0}\right)^{\gamma}}{\Gamma_{r}(\gamma+1)}\right) \Theta \| u \\
& -v\left\|_{\mathscr{X}}=\mathscr{L} \Theta\right\| u-v \|_{\mathscr{X}},
\end{aligned}
$$

where $\mathscr{L}$ and $\Theta$ are defined on (49)-(50), respectively.

$\left(\mathrm{H}_{2}\right)$ implies that $\mathscr{F}$ is a contraction. Therefore, by using Banach fixed-point theorem, $\mathscr{F}$ has a fixed point which is a unique solution of problem (4).

We next show the existence of a solution to (4) by the following Schauder's fixed-point theorem.

Theorem 18. Suppose that $\left(H_{1}\right)$ holds. Then, problem (4) has at least one solution.

Proof. We organize the proof into three steps.

Step 1. Verify $\mathscr{F}$ map bounded sets into bounded sets in $B_{R}=$ $\left\{u \in \mathscr{X}:\|u\|_{\mathscr{X}} \leq R\right\}$. We set $\max _{t \in I_{q, \omega}^{T}}|F(t, 0,0)|=K$ and choose a constant

$$
R \geq \frac{K \Theta}{1-\mathscr{L} \Theta},
$$

where $\mathscr{L}$ and $\Theta$ are defined on (49)-(50), respectively. Denote that

$$
\begin{aligned}
|\mathcal{S}(t, u, 0)|= & \left|F\left(t, u(t), \Psi_{r, \phi}^{\gamma} u(t)\right)-F(t, 0,0)\right| \\
& +|F(t, 0,0)| .
\end{aligned}
$$

For each $t \in I_{q, \omega}^{T}$ and $u \in B_{R}$, we obtain

$$
\begin{aligned}
& \mid \mathscr{P}^{*} {\left[F_{u}\right] \mid=\frac{1}{\Gamma_{q}(\alpha) \Gamma_{p}(-\beta)} } \\
& \cdot \int_{\omega_{0}}^{p T+\theta} \int_{\omega_{0}}^{x}\left(p T+\theta-\sigma_{p, \theta}(x)\right)_{p, \theta}^{\frac{-\beta-1}{p}} \\
& \quad \times\left(x-\sigma_{q, \omega}(s)\right)_{q, \omega}^{\frac{\alpha-1}{}}|\mathcal{S}(t, u, 0)| d_{q, \omega} s d_{p, \theta} x \\
& \leq\left[\left(\lambda_{1}+\frac{\lambda_{2} \varphi_{0}}{\Gamma_{r}(\gamma+1)}\left(T-\omega_{0}\right) \frac{\gamma+1}{r, \phi}\right)\|u\|_{\mathscr{X}}+K\right] \\
& \quad \cdot \frac{\Gamma_{q}(-\beta)\left(p\left(T-\omega_{0}\right)\right)^{\alpha-\beta}}{\Gamma_{p}(-\beta) \Gamma_{q}(\alpha-\beta+1)},
\end{aligned}
$$




$$
\begin{aligned}
& \left|Q^{*}\left[F_{u}\right]\right|=\frac{1}{\Gamma_{q}(\alpha)} \\
& \quad \cdot \int_{\omega_{0}}^{T}\left(T-\sigma_{q, \omega}(s)\right)_{q, \omega}^{\frac{\alpha-1}{2}}|\mathcal{S}(t, u, 0)| d_{q, \omega} s \\
& \leq\left[\left(\lambda_{1}+\frac{\lambda_{2} \varphi_{0}}{\Gamma_{r}(\gamma+1)}\left(T-\omega_{0}\right) \frac{\gamma+1}{r, \phi}\right)\|u\|_{X}+K\right] \\
& \quad \cdot \frac{\left(T-\omega_{0}\right)^{\alpha}}{\Gamma_{q}(\alpha+1)} .
\end{aligned}
$$

Similarly with Theorem 17, we obtain

$$
\begin{aligned}
& |(\mathscr{F} u)(t)| \\
& \quad \leq\left[\left(\lambda_{1}+\frac{\lambda_{2} \varphi_{0}}{\Gamma_{r}(\gamma+1)}\left(T-\omega_{0}\right) \frac{\gamma+1}{r, \phi}\right)\|u\|_{\mathscr{X}}+K\right] \Theta \\
& \quad=\left[\mathscr{L}\|u\|_{\mathscr{X}}+K\right] \Theta \leq[\mathscr{L} R+K] \Theta \leq R .
\end{aligned}
$$

Therefore $\|\mathscr{F} u\|_{\mathscr{X}} \leq R$. This implies that $\mathscr{F}$ is uniformly bounded.

Step 2. Show that $\mathscr{F}$ is continuous on $B_{R}$.

Letting $\epsilon>0$, there exists $\delta>0$ such that, for each $t \in I_{q, \omega}^{T}$ and for all $u, v \in B_{R}$,

$$
\mathscr{H}|u-v|<\frac{\epsilon}{\Theta} \quad \text { whenever }|u-v|<\delta
$$

Then, we have

$$
\|\mathscr{F} u-\mathscr{F} v\|_{\mathscr{X}} \leq \mathscr{H}\|u-v\| \Theta<\epsilon .
$$

This means that the operator $\mathscr{F}$ is continuous on $B_{R}$.

Step 3. Examine that $\mathscr{F}$ is equicontinuous with $B_{R}$. For any $t_{1}, t_{2} \in I_{q, \omega}^{T}$ with $t_{1}<t_{2}$, we have

$$
\begin{aligned}
& \left|(\mathscr{F} u)\left(t_{2}\right)-(\mathscr{F} u)\left(t_{1}\right)\right| \leq \mid \frac{\left(t_{2}-\omega_{0}\right)^{\alpha-1}}{\Omega} \\
& -\frac{\left(t_{1}-\omega_{0}\right)^{\alpha-1}}{\Omega}||\left(T-\omega_{0}\right)^{\alpha-2} \mathscr{P}^{*}\left[F_{u}\right] \\
& -\Lambda_{2} Q^{*}\left[F_{u}\right]|+| \frac{\left(t_{2}-\omega_{0}\right)^{\alpha-2}}{\Omega}-\frac{\left(t_{1}-\omega_{0}\right)^{\alpha-2}}{\Omega} \mid \\
& \cdot\left|\left(T-\omega_{0}\right)^{\alpha-1} \mathscr{P}^{*}\left[F_{u}\right]-\Lambda_{1} Q^{*}\left[F_{u}\right]\right| \\
& +\frac{\|F\|}{\Gamma_{q}(\alpha)} \mid \int_{\omega_{0}}^{t_{2}}\left(t_{2}-\sigma_{q, \omega}(s)\right)_{q, \omega}^{\frac{\alpha-1}{q}} d_{q, \omega} s \\
& -\int_{\omega_{0}}^{t_{1}}\left(t_{1}-\sigma_{q, \omega}(s)\right)_{q, \omega}^{\frac{\alpha-1}{\omega}} d_{q, \omega} s \mid .
\end{aligned}
$$

If $\left|t_{2}-t_{1}\right| \rightarrow 0$, the right-hand side of the above inequality tends to be zero. So $\mathscr{F}$ is relatively compact on $B_{R}$.

This means that the set $\mathscr{F}\left(B_{R}\right)$ is an equicontinuous set. As a consequence of Steps I to III together with the Arzelá-Ascoli theorem, we get that $\mathscr{F}: \mathscr{X} \rightarrow \mathscr{X}$ is completely continuous. By Schauder's fixed-point theorem, we can conclude that problem (4) has at least one solution. The proof is done.

\section{Existence Results for Problem (5)}

In this section, we present the existence results for problem (5). Let $\mathcal{Y}=C\left(I_{q, \omega}^{T}, \mathbb{R}\right)$ be a Banach space of all function $u$ with the norm defined by

$$
\|u\|_{y}=\max _{t \in I_{q, \omega}^{T}}\left\{|u(t)|,\left|{ }^{C} D_{r, \phi}^{\gamma} u(r t+\phi)\right|\right\},
$$

where $\omega>0, p, q, r \in(0,1), p=q^{m}, r=q^{n}, m, n \in \mathbb{N}$, $\theta=\omega((1-p) /(1-q)), \phi=\omega((1-r) /(1-q))$, and $\mathscr{A}, \mathscr{B}$ : $C\left(I_{q, \omega}^{T}, \mathbb{R}\right) \rightarrow \mathbb{R}$. Define an operator $\mathscr{G}: \mathcal{Y} \rightarrow \mathcal{Y}$ by

$$
\begin{aligned}
& (\mathscr{G} u)(t):=\mathscr{A}(u)+\frac{\left(t-\omega_{0}\right) \Gamma_{q}(\beta+2)}{\left(T-\omega_{0}\right)^{\beta+1}}\{\mathscr{B}(u) \\
& -\frac{\left(T-\omega_{0}\right)^{\beta}}{\Gamma_{q}(\beta+1)} \mathscr{A}(u)-\frac{1}{\Gamma_{q}(\alpha) \Gamma_{p}(\beta)} \\
& \times \int_{\omega_{0}}^{T} \int_{\omega_{0}}^{x}\left(T-\sigma_{p, \theta}(x)\right)_{p, \theta}^{\beta-1}\left(x-\sigma_{q, \omega}(s)\right)_{q, \omega}^{\frac{\alpha-1}{2}} \\
& \left.\cdot G\left(t, u(t),{ }^{C} D_{r, \phi}^{\gamma} u(r t+\phi)\right) d_{q, \omega} s d_{p, \theta} x\right\} \\
& +\frac{1}{\Gamma_{q}(\alpha)} \int_{\omega_{0}}^{t}\left(t-\sigma_{q, \omega}(s)\right)_{q, \omega}^{\frac{\alpha-1}{\omega}} G\left(t, u(t),{ }^{C} D_{r, \phi}^{\gamma} u(r t\right. \\
& +\phi)) d_{q, \omega} s .
\end{aligned}
$$

Obviously, problem (5) has solutions if and only if the operator $\mathscr{G}$ has fixed points.

Theorem 19. Assume that $G: I_{q, \omega}^{T} \times \mathbb{R} \times \mathbb{R} \rightarrow \mathbb{R}$ is continuous and $\mathscr{A}, \mathscr{B}: C\left(I_{q, \omega}^{T}, \mathbb{R}\right) \rightarrow \mathbb{R}$. In addition, suppose that the following conditions hold:

$\left(H_{3}\right)$ There exist constants $\mu_{1}, \mu_{2}>0$ such that, for each $t \in I_{q, \omega}^{T}$ and $u, v \in \mathbb{R}$,

$$
\begin{aligned}
& \left|G\left(t, u,{ }^{C} D_{r, \phi}^{\gamma} u\right)-G\left(t, v,{ }^{C} D_{r, \phi}^{\gamma} v\right)\right| \\
& \quad \leq \mu_{1}|u-v|+\mu_{2}\left|{ }^{C} D_{r, \phi}^{\gamma} u-{ }^{C} D_{r, \phi}^{\gamma} v\right| .
\end{aligned}
$$

$\left(H_{4}\right)$ There exist constants $\ell_{1}, \ell_{2}>0$ such that, for each $u, v \in$ $y$,

$$
\begin{gathered}
|\mathscr{A}(u)-\mathscr{A}(v)| \leq \ell_{1}\|u-v\|_{y}, \\
|\mathscr{B}(u)-\mathscr{B}(v)| \leq \ell_{2}\|u-v\|_{y} .
\end{gathered}
$$


$\left(H_{5}\right) \Phi<1$, where

$$
\begin{aligned}
\Phi:=(1 & \left.+[\beta+1]_{q}\right) \ell_{1}+\frac{\Gamma_{q}(\beta+2)}{\left(T-\omega_{0}\right)^{\beta}} \ell_{2}+\left(\mu_{1}+\mu_{2}\right) \\
& \cdot\left(T-\omega_{0}\right)^{\alpha} \\
& \cdot\left\{\frac{\Gamma_{q}(\beta) \Gamma_{q}(\beta+2)}{\Gamma_{p}(\beta) \Gamma_{q}(\alpha+\beta+1)}+\frac{1}{\Gamma_{q}(\alpha+1)}\right\} .
\end{aligned}
$$

Then, problem (5) has a unique solution.

Proof. To show that $F$ is contraction, we consider

$$
\begin{aligned}
\mathscr{K} & |u-v|(t):=\mid G\left(t, u(t),{ }^{C} D_{r, \phi}^{\gamma} u(r t+\phi)\right) \\
& -G\left(t, v(t),{ }^{C} D_{r, \phi}^{\gamma} v(r t+\phi)\right) \mid \\
& \leq \| G\left(t, u(t),{ }^{C} D_{r, \phi}^{\gamma} u(r t+\phi)\right) \\
& -G\left(t, v(t),{ }^{C} D_{r, \phi}^{\gamma} v(r t+\phi)\right)\|=: \mathscr{K}\| u-v \|,
\end{aligned}
$$

for each $t \in I_{q, \omega}^{T}$ and $u, v \in \mathcal{Y}$. that

For each $t \in I_{q, \omega}^{T}$ and $u, v,{ }^{C} D_{r, \phi}^{\gamma} u,{ }^{C} D_{r, \phi}^{\gamma} v \in \mathcal{Y}$, we find

$$
\begin{aligned}
& |(\mathscr{G} u)(t)-(\mathscr{G} v)(t)| \leq\left[1+\frac{\left(t-\omega_{0}\right)[\beta+1]_{q}}{\left(T-\omega_{0}\right)}\right] \\
& \cdot|\mathscr{A}(u)-\mathscr{A}(v)|+\frac{\left(t-\omega_{0}\right) \Gamma_{q}(\beta+2)}{\left(T-\omega_{0}\right)^{\beta+1}} \mid \mathscr{B}(u) \\
& -\mathscr{B}(v) \mid+\frac{\left(t-\omega_{0}\right) \Gamma_{q}(\beta+2)}{\left(T-\omega_{0}\right)^{\beta+1} \Gamma_{q}(\alpha) \Gamma_{p}(\beta)} \int_{\omega_{0}}^{T} \int_{\omega_{0}}^{x}(T \\
& \left.-\sigma_{p, \theta}(x)\right)_{p, \theta}^{\beta-1}\left(x-\sigma_{q, \omega}(s)\right)_{q, \omega}^{\frac{\alpha-1}{K}|u-v|} \\
& \cdot(s) d_{q, \omega} s d_{p, \theta} x+\frac{1}{\Gamma_{q}(\alpha)} \\
& \cdot \int_{\omega_{0}}^{t}\left(t-\sigma_{q, \omega}(s)\right)_{q, \omega}^{\frac{\alpha-1}{K}|u-v|(s) d_{q, \omega} s \leq(1} \\
& \left.+[\beta+1]_{q}\right) \ell_{1}\|u-v\|_{\mathscr{Y}}+\frac{\Gamma_{q}(\beta+2)}{\left(T-\omega_{0}\right)^{\beta}} \ell_{2}\|u-v\|_{\mathscr{Y}} \\
& +\left(\mu_{1}|u(t)-v(t)|+\mu_{2}\left|D_{r, \phi}^{\gamma} u(t)-D_{r, \phi}^{\gamma} v(t)\right|\right) \\
& \quad+\left\{\frac{\Gamma_{q}(\beta+2)}{\left(T-\omega_{0}\right)^{\beta} \Gamma_{q}(\alpha) \Gamma_{p}(\beta)}\right. \\
& \quad \int_{\omega_{0}}^{T} \int_{\omega_{0}}^{x}\left(T-\sigma_{p, \theta}(x)\right)_{p, \theta}^{\beta-1}
\end{aligned}
$$

$$
\cdot\left(x-\sigma_{q, \omega}(s)\right)_{q, \omega}^{\alpha-1} d_{q, \omega} s d_{p, \theta} x+\frac{1}{\Gamma_{q}(\alpha)}
$$$$
\left.\cdot \int_{\omega_{0}}^{T}\left(T-\sigma_{q, \omega}(s)\right)_{q, \omega}^{\frac{\alpha-1}{q}} d_{q, \omega} s\right\} \leq\|u-v\|_{y}
$$$$
\cdot\left\{\left(1+[\beta+1]_{q}\right) \ell_{1}+\frac{\Gamma_{q}(\beta+2)}{\left(T-\omega_{0}\right)^{\beta}} \ell_{2}\right\}+\|u-v\|_{y}
$$$$
\cdot\left(\mu_{1}+\mu_{2}\right)\left(T-\omega_{0}\right)^{\alpha}\left\{\frac{\Gamma_{q}(\beta) \Gamma_{q}(\beta+2)}{\Gamma_{p}(\beta) \Gamma_{q}(\alpha+\beta+1)}\right.
$$$$
\left.+\frac{1}{\Gamma_{q}(\alpha+1)}\right\} \leq\|u-v\|_{\mathscr{Y}} \Phi,
$$$$
\left|\left({ }^{C} D_{r, \phi}^{\gamma} \mathscr{G} u\right)(r t+\phi)-\left({ }^{C} D_{r, \phi}^{\gamma} \mathscr{G} v\right)(r t+\phi)\right| \leq[1
$$$$
\left.+\frac{[\beta+1]_{q} C}{\left(T-\omega_{0}\right)} D_{r, \phi}^{\gamma} r\left(t-\omega_{0}\right)\right]|\mathscr{A}(u)-\mathscr{A}(v)|
$$$$
+\frac{\Gamma_{q}(\beta+2)}{\left(T-\omega_{0}\right)^{\beta+1}}|\mathscr{B}(u)-\mathscr{B}(v)|{ }^{C} D_{r, \phi}^{\gamma} r\left(t-\omega_{0}\right)
$$$$
+\frac{\Gamma_{q}(\beta+2)^{C} D_{r, \phi}^{\gamma} r\left(t-\omega_{0}\right)}{\left(T-\omega_{0}\right)^{\beta+1} \Gamma_{q}(\alpha) \Gamma_{p}(\beta)} \int_{\omega_{0}}^{T} \int_{\omega_{0}}^{x}(T
$$$$
\left.-\sigma_{p, \theta}(x)\right)_{p, \theta}^{\frac{\beta-1}{}}\left(x-\sigma_{q, \omega}(s)\right)_{q, \omega}^{\frac{\alpha-1}{K}} \mathscr{K}|u-v|
$$$$
\text { . (s) } d_{q, \omega} s d_{p, \theta} x+\frac{1}{\Gamma_{q}(\alpha)}
$$$$
\cdot{ }^{C} D_{r, \phi}^{\gamma}\left[\int_{\omega_{0}}^{r t+\phi}\left(r t+\phi-\sigma_{q, \omega}(s)\right)_{q, \omega}^{\frac{\alpha-1}{K}|u-v|}\right.
$$$$
\left.\cdot(s) d_{q, \omega} s\right] \leq[1
$$$$
\left.+\frac{r^{1-\gamma}[\beta+1]_{q}\left(T-\omega_{0}\right)^{-\gamma}}{\Gamma_{r}(2-\gamma)}\right] \ell_{1}\|u-v\|_{y}
$$$$
+\left[\frac{r^{1-\gamma} \Gamma_{q}(\beta+2)\left(T-\omega_{0}\right)^{\beta-\gamma}}{\Gamma_{r}(2-\gamma)}\right] \ell_{2}\|u-v\|_{y}
$$$$
+\frac{r^{1-\gamma} \Gamma_{q}(\beta)\left(\Gamma_{q}(\beta+2)\right)^{2}\left(T-\omega_{0}\right)^{\alpha+\beta-\gamma}}{\Gamma_{q}(\alpha)\left(\Gamma_{p}(\beta)\right)^{2} \Gamma_{q}(\alpha+\beta+1) \Gamma_{r}(2-\gamma)} \cdot \| u
$$$$
-v \|_{y}\left(\mu_{1}+\mu_{2}\right)
$$$$
+\frac{r^{\alpha-\gamma} \Gamma_{r}(\alpha+1)\left(T-\omega_{0}\right)^{\alpha-\gamma}}{[1-\gamma]_{r} \Gamma_{q}(\alpha+1) \Gamma_{r}(\alpha-\gamma+1)} \cdot\|u-v\|_{\mathscr{Y}}\left(\mu_{1}\right.
$$$$
\left.+\mu_{2}\right)<\|u-v\|_{y} \Phi \text {. }
$$ 
Hence, from (67), it is implied that $\|(\mathscr{G} u)(t)-(\mathscr{G} v)(t)\|_{\mathscr{Y}} \leq$ $\|u-v\|_{y} \Phi$.

By $\left(H_{5}\right)$, it is implied that $\mathscr{G}$ is a contraction. Therefore, by using Banach fixed-point theorem, $\mathscr{G}$ has a fixed point which is a unique solution of problem (5).

We also deduce the existence of a solution to (5) by the following Schauder's fixed-point theorem.
Theorem 20. Suppose that $\left(H_{3}\right)-\left(H_{4}\right)$ hold. Then, problem (5) has at least one solution.

Proof. We divide the proof into three steps.

Step 1 . Verify $\mathscr{G}$ map bounded sets into bounded sets in $B_{L}=$ $\left\{u \in \mathcal{Y}:\|u\|_{\mathscr{Y}} \leq L\right\}$. We set $\max _{t \in I_{q, \omega}^{T}}|G(t, 0,0)|=K$,

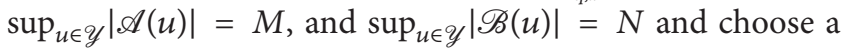
constant

$L$

$$
\leq \frac{\left(1+[\beta+1]_{q}\right) M+\left(\Gamma_{q}(\beta+2) /\left(T-\omega_{0}\right)^{\beta}\right) N+K\left(\mu_{1}+\mu_{2}\right)\left(T-\omega_{0}\right)^{\alpha}\left[\Gamma_{q}(\beta) \Gamma_{q}(\beta+2) / \Gamma_{p}(\beta) \Gamma_{q}(\alpha+\beta+1)+1 / \Gamma_{q}(\alpha+1)\right]}{1-\Phi},
$$

where $\Phi$ are defined on (65). Denote that

$$
\begin{aligned}
|\mathcal{T}(t, u, 0)| \\
=\left|G\left(t, u(t),{ }^{C} D_{r, \phi}^{\gamma} u(r t+\phi)\right)-G(t, 0,0)\right| \\
\quad+|G(t, 0,0)| .
\end{aligned}
$$

For each $t \in I_{q, \omega}^{T}$ and $u \in B_{L}$, we obtain

$$
\begin{aligned}
& |(\mathscr{G} u)(t)| \leq\left[1+\frac{\left(t-\omega_{0}\right)[\beta+1]_{q}}{\left(T-\omega_{0}\right)}\right]|\mathscr{A}(u)| \\
& +\frac{\left(t-\omega_{0}\right) \Gamma_{q}(\beta+2)}{\left(T-\omega_{0}\right)^{\beta+1}}|\mathscr{B}(u)| \\
& +\frac{\left(t-\omega_{0}\right) \Gamma_{q}(\beta+2)}{\left(T-\omega_{0}\right)^{\beta+1} \Gamma_{q}(\alpha) \Gamma_{p}(\beta)} \int_{\omega_{0}}^{T} \int_{\omega_{0}}^{x}(T \\
& \left.-\sigma_{p, \theta}(x)\right)_{p, \theta}^{\beta-1}\left(x-\sigma_{q, \omega}(s)\right)_{q, \omega}^{\frac{\alpha-1}{T}} \mathscr{T}|u-v| \\
& \cdot(s) d_{q, \omega} s d_{p, \theta} x+\frac{1}{\Gamma_{q}(\alpha)} \\
& \cdot \int_{\omega_{0}}^{t}\left(t-\sigma_{q, \omega}(s)\right)_{q, \omega}^{\frac{\alpha-1}{T}} \mathscr{T}|u-v|(s) d_{q, \omega} s \leq(1 \\
& \left.+[\beta+1]_{q}\right)\left(\ell_{1}\|u\|_{\mathscr{Y}}+M\right)+\frac{\Gamma_{q}(\beta+2)}{\left(T-\omega_{0}\right)^{\beta}}\left(\ell_{2}\|u\|_{\mathscr{Y}}\right. \\
& +N)+\left(\mu_{1}|u(t)|+\mu_{2}\left|D_{r, \phi}^{\gamma} u(t)\right|+K\right) \\
& \text {. }\left\{\frac{\Gamma_{q}(\beta+2)}{\left(T-\omega_{0}\right)^{\beta} \Gamma_{q}(\alpha) \Gamma_{p}(\beta)}\right.
\end{aligned}
$$

$$
\begin{aligned}
& \times \int_{\omega_{0}}^{T} \int_{\omega_{0}}^{x}\left(T-\sigma_{p, \theta}(x)\right)_{p, \theta}^{\beta-1} \\
& \cdot\left(x-\sigma_{q, \omega}(s)\right)_{q, \omega}^{\frac{\alpha-1}{q}} d_{q, \omega} s d_{p, \theta} x+\frac{1}{\Gamma_{q}(\alpha)} \\
& \left.\cdot \int_{\omega_{0}}^{T}\left(T-\sigma_{q, \omega}(s)\right)_{q, \omega}^{\frac{\alpha-1}{q}} d_{q, \omega} s\right\} \leq\|u\|_{y}\{(1 \\
& \left.\left.+[\beta+1]_{q}\right) \ell_{1}+\frac{\Gamma_{q}(\beta+2)}{\left(T-\omega_{0}\right)^{\beta}} \ell_{2}\right\}+\left(1+[\beta+1]_{q}\right) M \\
& +\frac{\Gamma_{q}(\beta+2)}{\left(T-\omega_{0}\right)^{\beta}} N+\left(\|u\|_{y}+K\right)\left(\mu_{1}+\mu_{2}\right)\left(T-\omega_{0}\right)^{\alpha} \\
& \cdot\left\{\frac{\Gamma_{q}(\beta) \Gamma_{q}(\beta+2)}{\Gamma_{p}(\beta) \Gamma_{q}(\alpha+\beta+1)}+\frac{1}{\Gamma_{q}(\alpha+1)}\right\} \leq L \Phi+(1 \\
& \left.+[\beta+1]_{q}\right) M+\frac{\Gamma_{q}(\beta+2)}{\left(T-\omega_{0}\right)^{\beta}} N+K\left(\mu_{1}+\mu_{2}\right)(T \\
& \left.-\omega_{0}\right)^{\alpha}\left\{\frac{\Gamma_{q}(\beta) \Gamma_{q}(\beta+2)}{\Gamma_{p}(\beta) \Gamma_{q}(\alpha+\beta+1)}+\frac{1}{\Gamma_{q}(\alpha+1)}\right\} \leq L .
\end{aligned}
$$

Similar to Theorem 19, we obtain

$$
\left|\left({ }^{C} D_{r, \phi}^{\gamma} \mathscr{G} u\right)(r t+\phi)\right|<L .
$$

So, we have $\|\mathscr{G} u\|_{\mathscr{Y}} \leq L$. This implies that $\mathscr{G}$ is uniformly bounded.

Step 2. Show that $\mathscr{G}$ is continuous on $B_{L}$.

Letting $\epsilon>0$, there exist $\delta=\max \left\{\delta_{1}, \delta_{2}, \delta_{3}\right\}>0$ such that, for each $t \in I_{q, \omega}^{T}$ and for all $u, v \in B_{L}$,

$$
\mathscr{K}|u-v|<\frac{\epsilon}{3\left(T-\omega_{0}\right)^{\alpha}\left[\Gamma_{q}(\beta) \Gamma_{q}(\beta+2) / \Gamma_{p}(\beta) \Gamma_{q}(\alpha+\beta+1)+1 / \Gamma_{q}(\alpha+1)\right]} \quad \text { whenever }|u-v|<\delta_{1}
$$




$$
\begin{gathered}
|\mathscr{A}(u)-\mathscr{A}(v)|<\frac{\epsilon}{3\left(1+[\beta+1]_{q}\right)} \quad \text { whenever }|u-v|<\delta_{2}, \\
|\mathscr{B}(u)-\mathscr{B}(v)|<\frac{\epsilon\left(T-\omega_{0}\right)^{\beta}}{3 \Gamma_{q}(\beta+2)} \quad \text { whenever }|u-v|<\delta_{3} .
\end{gathered}
$$

Then, we have

$$
\begin{aligned}
\|\mathscr{G} u-\mathscr{G} v\| \leq\left(1+[\beta+1]_{q}\right)\|\mathscr{A}(u)-\mathscr{A}(v)\| \\
+\left[\frac{\Gamma_{q}(\beta+2)}{\left(T-\omega_{0}\right)^{\beta}}\right]\|\mathscr{B}(u)-\mathscr{B}(v)\|+\left(T-\omega_{0}\right)^{\alpha} \\
+\left[\frac{\Gamma_{q}(\beta) \Gamma_{q}(\beta+2)}{\Gamma_{p}(\beta) \Gamma_{q}(\alpha+\beta+1)}+\frac{1}{\Gamma_{q}(\alpha+1)}\right] \mathscr{K}\|u-v\| \\
<\frac{\epsilon}{3}+\frac{\epsilon}{3}+\frac{\epsilon}{3}=\epsilon .
\end{aligned}
$$

By a similar proof to the above, we obtain

$$
\left\|{ }^{C} D_{r, \phi}^{\gamma} \mathscr{G}-{ }^{C} D_{r, \phi}^{\gamma} \mathscr{G} v\right\|<\epsilon .
$$

Hence, $\|\mathscr{G} u-\mathscr{G} v\|_{\mathscr{Y}}<\epsilon$. This means that the operator $\mathscr{G}$ is continuous on $B_{L}$.

Step 3. Examine that $\mathscr{G}$ is equicontinuous with $B_{L}$. For any $t_{1}, t_{2} \in I_{q, \omega}^{T}$ with $t_{1}<t_{2}$,

$$
\begin{aligned}
& \left|(\mathscr{G} u)\left(t_{2}\right)-(\mathscr{G} u)\left(t_{1}\right)\right| \leq\left|t_{2}-t_{1}\right| \\
& \cdot \frac{\Gamma_{q}(\beta+2)}{\left(T-\omega_{0}\right)^{\beta+1}}\left\{\mathscr{B}(u)-\frac{\left(T-\omega_{0}\right)^{\beta}}{\Gamma_{q}(\beta+1)} \mathscr{A}(u)\right. \\
& -\frac{\|G\|}{\Gamma_{q}(\alpha) \Gamma_{p}(\beta)} \times \int_{\omega_{0}}^{T} \int_{\omega_{0}}^{x}\left(T-\sigma_{p, \theta}(x)\right)_{p, \theta}^{\beta-1} \\
& \left.\cdot\left(x-\sigma_{q, \omega}(s)\right)_{q, \omega}^{\frac{\alpha-1}{q}} d_{q, \omega} s d_{p, \theta} x\right\} \\
& +\frac{\|G\|}{\Gamma_{q}(\alpha)} \mid \int_{\omega_{0}}^{t_{2}}\left(t_{2}-\sigma_{q, \omega}(s)\right)_{q, \omega}^{\frac{\alpha-1}{q, \omega}} d_{q-\int_{\omega_{0}}}^{t_{1}}\left(t_{1}\right. \\
& \left.-\sigma_{q, \omega}(s)\right)_{q, \omega}^{\frac{\alpha-1}{q}} d_{q, \omega} s \mid, \\
& \mid\left({ }^{C} D_{r, \phi}^{\gamma} \mathscr{G}_{u} u\left(r t_{2}+\phi\right)-\left({ }^{C} D_{r, \phi}^{\gamma} \mathscr{G}_{u}\right)\left(r t_{1}+\phi\right) \mid\right. \\
& \quad \leq{ }^{C} D_{r, \phi}^{\gamma}\left|t_{2}-t_{1}\right| \frac{\Gamma_{q}(\beta+2)}{\left(T-\omega_{0}\right)^{\beta+1}}\left\{\mathscr{B}(u)-\frac{\left(T-\omega_{0}\right)^{\beta}}{\Gamma_{q}(\beta+1)}\right. \\
& \cdot \mathscr{A}(u)-\frac{\|G\|}{\Gamma_{q}(\alpha) \Gamma_{p}(\beta)} \times \int_{\omega_{0}}^{T} \int_{\omega_{0}}^{x}\left(T-\sigma_{p, \theta}(x)\right)_{p, \theta}^{\beta-1} \\
& \left.\cdot\left(x-\sigma_{q, \omega}(s)\right)_{q, \omega}^{\alpha-1} d_{q, \omega} s d_{p, \theta} x\right\}
\end{aligned}
$$

$$
\begin{aligned}
& +\frac{\|G\|}{\Gamma_{q}(\alpha)}{ }^{C} D_{r, \phi}^{\gamma} \mid \int_{\omega_{0}}^{t_{2}}\left(t_{2}-\sigma_{q, \omega}(s)\right)_{q, \omega}^{\frac{\alpha-1}{q}} d_{q, \omega} s-\int_{\omega_{0}}^{t_{1}}\left(t_{1}\right. \\
& \left.-\sigma_{q, \omega}(s)\right)_{q, \omega}^{\frac{\alpha-1}{q}} d_{q, \omega} s \mid .
\end{aligned}
$$

If $\left|t_{2}-t_{1}\right| \rightarrow 0$, the right-hand side of the above inequalities (75) tends to be zero. So $\mathscr{G}$ is relatively compact on $B_{L}$.

Therefore the set $\mathscr{G}\left(B_{L}\right)$ is an equicontinuous set. As a consequence of Steps I to III together with the Arzelá-Ascoli theorem, we get that $\mathscr{G}: \mathcal{Y} \rightarrow \mathscr{Y}$ is completely continuous. By Schauder's fixed-point theorem, we can conclude that problem (5) has at least one solution. The proof is completed.

\section{Some Examples}

In this section, we provide some examples to illustrate our results.

Example 1. Consider the following fractional Hahn boundary value problem:

$$
\begin{aligned}
& D_{1 / 2,2}^{4 / 3} u(t) \\
& =\frac{e^{-2 t}|u|}{(t+2)^{3}(|u|+1)} \\
& \quad+\frac{\arctan \left(\sin ^{2} \pi t\right)}{10 e^{t} \Gamma_{1 / 8}(1 / 3)} \int_{4}^{t}\left(t-\sigma_{1 / 8,7 / 2}(s)\right)_{1 / 8,7 / 2}^{-2 / 3} \\
& \quad \times\left[\frac{e^{-3|s-t|}}{100+\cos ^{2} \pi t}\right] u(s) d_{1 / 8,7 / 2} s, \\
& \qquad t \in[4,10]_{1 / 22} \\
& u(4)=u(10), \\
& D_{1 / 4,3}^{1 / 2} u(4)=D_{1 / 4,3}^{1 / 2} u\left(\frac{11}{2}\right) .
\end{aligned}
$$

Here $\alpha=4 / 3, \beta=1 / 2, \gamma=1 / 3, q=1 / 2, p=1 / 4, r=1 / 8$, $\omega=2, \theta=3, \phi=7 / 2, \omega_{0}=\omega /(1-q)=4, T=10, \varphi(t, s)=$ $e^{-3|s-t|} /\left(100+\cos ^{2} \pi t\right), \varphi_{0}=1 / 200$, and $F\left(t, u, \Psi_{1 / 8,7 / 2}^{1 / 3} u\right)=$ $e^{-2 t}|u| /(t+2)^{3}(|u|+1)+\left(\arctan \left(\sin ^{2} \pi t\right) / 10 e^{t} \Gamma_{1 / 8}(1 / 3)\right) \int_{4}^{t}(t-$ $\left.\sigma_{1 / 8,7 / 2}(s)\right)_{1 / 8,7 / 2}^{-2 / 3} \varphi(t, s) u(s) d_{1 / 8,7 / 2} s$. 
Since

$$
\begin{aligned}
& \left|F\left(t, u, \Psi_{1 / 8,7 / 2}^{1 / 3} u\right)-F\left(t, v, \Psi_{1 / 8,7 / 2}^{1 / 3} v\right)\right| \\
& \quad \leq \frac{1}{216 e^{8}}|x-y|+\frac{\pi}{400 e^{4}}\left|\Psi_{1 / 8,7 / 2}^{1 / 3} u-\Psi_{1 / 8,7 / 2}^{1 / 3} v\right|,
\end{aligned}
$$

then $\left(H_{1}\right)$ is satisfied with $\lambda_{1}=1 / 216 e^{8}$ and $\lambda_{2}=\pi / 400 e^{4}$.

Also, we have

$$
\begin{aligned}
& \Lambda_{1}=0.00402, \\
& \Lambda_{2}=-0.1387, \\
& |\Omega|=0.00184 .
\end{aligned}
$$

We can show that

$$
\begin{aligned}
\mathscr{L} & =0.00002858, \\
\Theta & =64.270 .
\end{aligned}
$$

Therefore, we find that

$$
\mathscr{L} \Theta \approx 0.00184<1 .
$$

Hence, by Theorem 17, problem (76) has a unique solution on $[4,10]_{1 / 2,2}$.

Example 2. Consider the following fractional Hahn boundary value problem:

$$
\begin{aligned}
{ }^{C} D_{1 / 2,2}^{4 / 3} u(t)= & \frac{e^{-\sin ^{2} \pi t}|u|}{100+e^{\cos ^{2}(\pi t / 2)}} \\
& \cdot \frac{|u(t)| t^{-2}+\left|{ }^{C} D_{1 / 8,7 / 2}^{1 / 3} u(t)\right|}{1+|u(t)|}, t \in[4,10]_{1 / 2,2}, \\
u(4)= & \frac{|u|}{125 e^{3}} \cos ^{2} \pi u, \\
\mathscr{J}_{1 / 4,3}^{1 / 2} u(10)= & \frac{|u|}{100 \pi^{2}} \sin ^{2} \pi u .
\end{aligned}
$$

Here $\alpha=4 / 3, \beta=1 / 2, \gamma=1 / 3, q=1 / 2, p=1 / 4$, $r=1 / 8, \omega=2, \theta=3, \phi=7 / 2, \omega_{0}=\omega /(1-q)=4, T=10$, $\mathscr{A}(u)=\left(|u| / 125 e^{3}\right) \cos ^{2} \pi u, \mathscr{B}(u)=\left(|u| / 100 \pi^{2}\right) \sin ^{2} \pi u$, and $F\left(t, u,{ }^{C} D_{1 / 8,7 / 2}^{1 / 3} u\right)=\left(e^{-\sin ^{2} \pi t}|u| /\left(100+e^{\cos ^{2}(\pi t / 2)}\right)\right) \cdot\left(\left(|u(t)| t^{-2}+\right.\right.$ $\left.\left.\left|{ }^{C} D_{1 / 8,7 / 2}^{1 / 3} u(t)\right|\right) /(1+|u(t)|)\right)$.

Since

$$
\begin{aligned}
& \left|F\left(t, u,{ }^{C} D_{1 / 8,7 / 2}^{1 / 3} u\right)-F\left(t, v,{ }^{C} D_{1 / 8,7 / 2}^{1 / 3} v\right)\right| \\
& \quad \leq \frac{1}{1616}|u-v|+\frac{1}{101}\left|{ }^{C} D_{1 / 8,7 / 2}^{1 / 3} u-{ }^{C} D_{1 / 8,7 / 2}^{1 / 3} v\right|, \\
& |\mathscr{A}(u)-\mathscr{A}(v)| \leq \frac{1}{125 e^{3}}\|u-v\|_{y}, \\
& |\mathscr{B}(u)-\mathscr{B}(v)| \leq \frac{1}{100 \pi^{2}}\|u-v\|_{y},
\end{aligned}
$$

then $\left(H_{3}\right)$ and $\left(H_{4}\right)$ are satisfied with $\mu_{1}=1 / 1616$ and $\mu_{2}=$ $1 / 101$ and $\ell_{1}=1 / 125 e^{3}$ and $\ell_{2}=1 / 100 \pi^{2}$.

Therefore, we get

$$
\Phi \approx 0.2135<1 .
$$

Hence, by Theorem 19, problem (81) has a unique solution on $[4,10]_{1 / 2,2}$.

\section{Conflicts of Interest}

The authors declare that there are no conflicts of interest regarding the publication of this paper.

\section{Acknowledgments}

This research was funded by King Mongkut's University of Technology North Bangkok (Contract no. KMUTNB-GOV60-76).

\section{References}

[1] M. H. Annaby and Z. S. Mansour, q-Fractional Calculus and Equations, Lecture Notes in Mathematics, Springer, Berlin, Germany, 2012.

[2] V. Kac and P. Cheung, Quantum Calculus, Springer, New York, NY, USA, 2002.

[3] D. L. Jagerman, Difference Equations with Applications to Queues, Dekker, New York, NY, USA, 2000.

[4] K. A. Aldwoah, A. B. Malinowska, and D. F. M. Torres, "The power quantum calculus and variational problems," Dynamics of Continuous, Discrete \& Impulsive Systems Series B: Applications \& Algorithms, vol. 19, no. 1-2, pp. 93-116, 2012.

[5] A. M. C. Cruz, N. Martins, and D. F. M. Torres, "Symmetric differentiation on time scales," Applied Mathematics Letters, vol. 26, no. 2, pp. 264-269, 2013.

[6] B. Cruz and M. C. Artur, Symmetric Quantum Calculus [Ph.D. thesis], Aveiro University, Aveiro, Portugal, 2012.

[7] G.-C. Wu and D. Baleanu, "New applications of the variational iteration method from differential equations to $q$-fractional difference equations," Advances in Difference Equations, vol. 2013, p. 21, 2013.

[8] J. Tariboon and S. K. Ntouyas, "Quantum calculus on finite intervals and applications to impulsive difference equations," Advances in Difference Equations, vol. 2013, article 282, 2013.

[9] R. Álvarez-Nodarse, "On characterizations of classical polynomials," Journal of Computational and Applied Mathematics, vol. 196, no. 1, pp. 320-337, 2006.

[10] W. Hahn, "Über Orthogonalpolynome, die q-Differenzenlgleichungen genügen," Mathematische Nachrichten, vol. 2, pp. 4-34, 1949.

[11] R. S. Costas-Santos and F. Marcellain, "Second structure Relation for q-semiclassical polynomials of the Hahn Tableau," Journal of Mathematical Analysis and Applications, vol. 329, pp. 206-228, 2007.

[12] K. H. Kwon, D. W. Lee, S. B. Park, and B. H. Yoo, "Hahn class orthogonal polynomials," Kyungpook Mathematical Journal, vol. 38 , no. 2, pp. 259-281, 1998.

[13] M. Foupouagnigni, Laguerre-Hahn orthogonal polynomials with respect to the Hahn operator: fourth-order difference equation for 
the rth associated and the Laguerre-Freud equations recurrence coefficients [Ph.D. thesis], Université Nationale du Bénin, Bénin, 1998.

[14] K. A. Aldwoah, Generalized time scales and associated difference equations [Ph.D. thesis], Cairo University, cairo, Egypt, 2009.

[15] M. H. Annaby, A. E. Hamza, and K. A. Aldwoah, "Hahn difference operator and associated Jackson-Nörlund integrals," Journal of Optimization Theory and Applications, vol. 154, no. 1, pp. 133-153, 2012.

[16] F. H. Jackson, "Basic integration," Quarterly Journal of Mathematics, vol. 2, pp. 1-16, 1951.

[17] A. B. Malinowska and D. F. Torres, "The Hahn quantum variational calculus," Journal of Optimization Theory and Applications, vol. 147, no. 3, pp. 419-442, 2010.

[18] A. B. Malinowska and D. F. Torres, "Quantum variational calculus," in Spinger Briefs in Electrical and Computer EngineeringControl, Automation and Robotics, Springer, Berlin, Germany, 2014.

[19] A. B. Malinowska and N. Martins, "Generalized transversality conditions for the Hahn quantum variational calculus," Optimization. A Journal of Mathematical Programming and Operations Research, vol. 62, no. 3, pp. 323-344, 2013.

[20] A. E. Hamza and S. M. Ahmed, "Existence and uniqueness of solutions of Hahn difference equations," Advances in Difference Equations, vol. 4, no. 2, pp. 441-461, 2013.

[21] A. E. Hamza and S. M. Ahmed, "Existence and uniqueness of solutions of Hahn difference equations," Advances in Difference Equations, vol. 2013, article 316, 2013.

[22] A. E. Hamza and S. D. Makharesh, "Leibniz' rule and Fubinis theorem associated with Hahn difference operator," Journal of Advanced Mathematical, vol. 12, no. 6, pp. 6335-6345, 2016.

[23] T. Sitthiwirattham, "On a nonlocal boundary value problem for nonlinear second-order Hahn difference equation with two different q, $\omega$-derivatives," Advances in Difference Equations, vol. 2016, no. 1, article no. 116, 2016.

[24] J. Čermák and L. Nechvátal, "On $(q, h)$-analogue of fractional calculus," Journal of Nonlinear Mathematical Physics, vol. 17, no. 1, pp. 51-68, 2010.

[25] J. Čermák, T. Kisela, and L. Nechvátal, "Discrete Mittag-Leffler functions in linear fractional difference equations," Abstract and Applied Analysis, vol. 2011, Article ID 565067, 21 pages, 2011.

[26] M. R. S. Rahmat, “The ( $q, h)$-Laplace transform on discrete time scales," Computers and Mathematics with Applications, vol. 62, no. 1, pp. 272-281, 2011.

[27] M. R. S. Rahmat, "On some ( $q, h)$-analogues of integral inequalities on discrete time scales," Computers and Mathematics with Applications, vol. 62, no. 4, pp. 1790-1797, 2011.

[28] F. Du, B. Jia, L. Erbe, and A. Peterson, "Monotonicity and convexity for nabla fractional $(q, h)$-difference," Journal of Difference Equations and Applications, vol. 22, no. 9, pp. 1224-1243, 2016.

[29] T. Brikshavana and T. Sitthiwirattham, "On fractional Hahn calculus," Advances in Difference Equations, vol. 2017, article 354, 2017.

[30] D. H. Griffel, Applied Functional Analysis, Ellis Horwood, Chichester, UK, 1981.

[31] D. J. Guo and V. Lakshmikantham, Nonlinear Problems in Abstract Cones, Academic Press, Orlando, Florida, Fla, USA, 1988. 


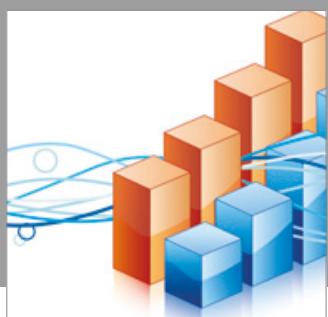

Advances in

Operations Research

vatersals

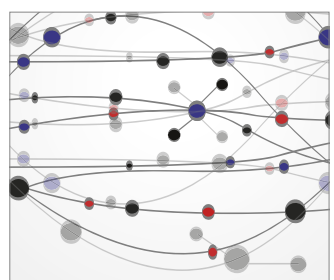

\section{The Scientific} World Journal
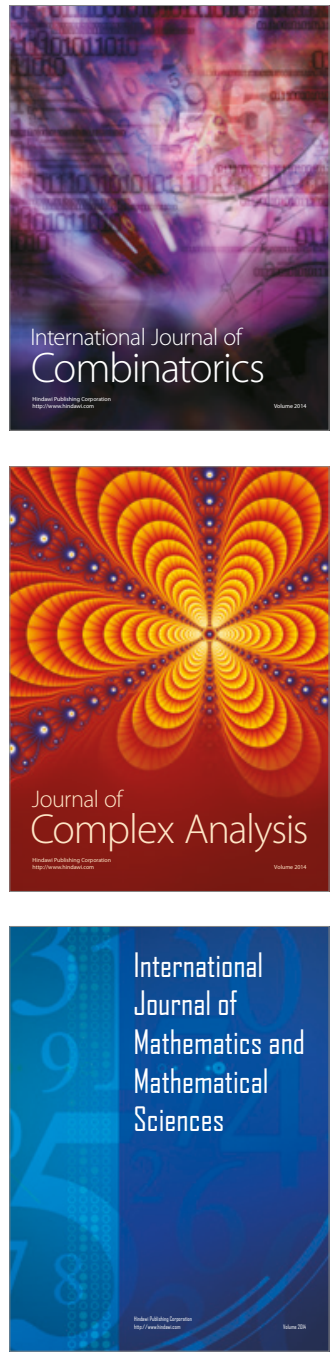
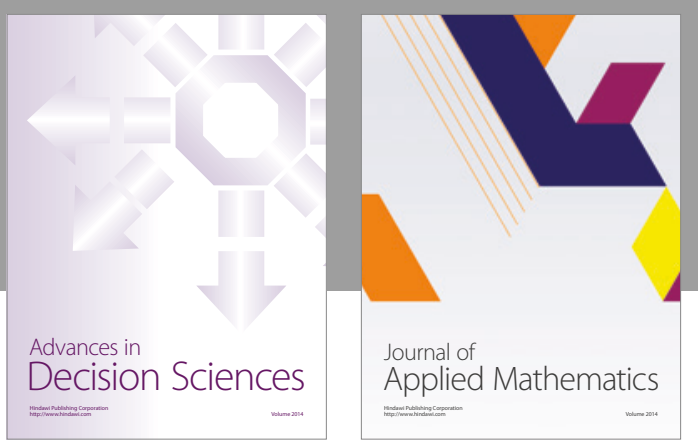

Algebra

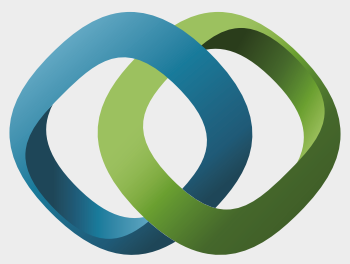

\section{Hindawi}

Submit your manuscripts at

https://www.hindawi.com
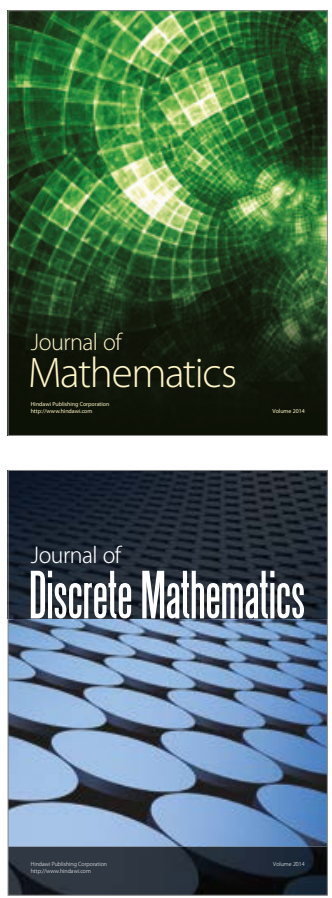

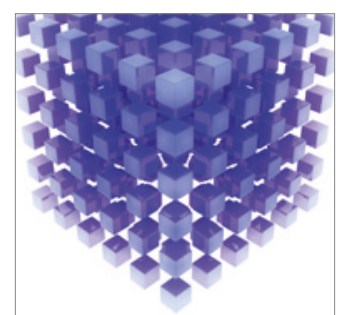

Mathematical Problems in Engineering
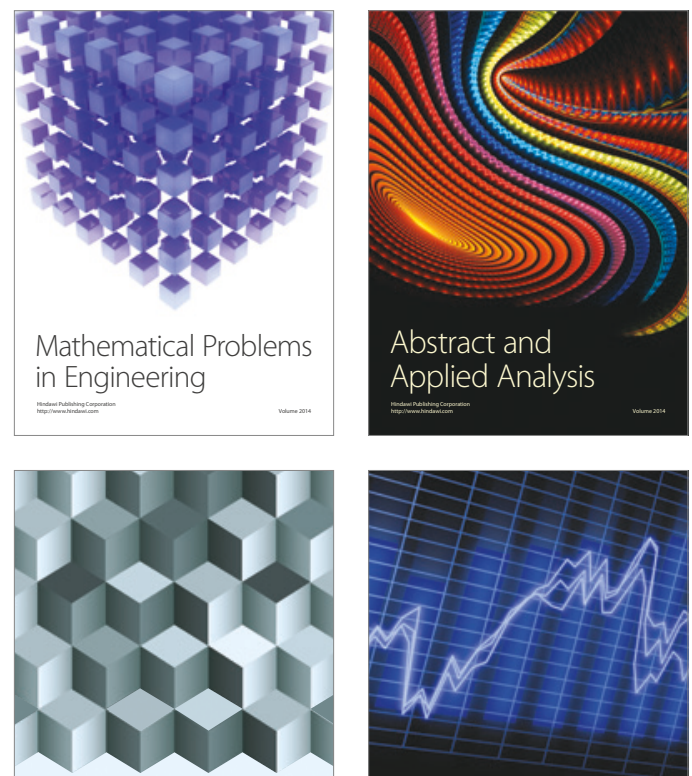

Journal of

Function Spaces

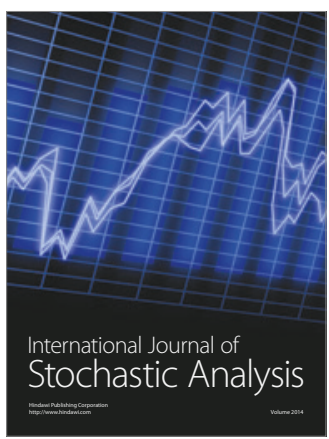

Probability and Statistics
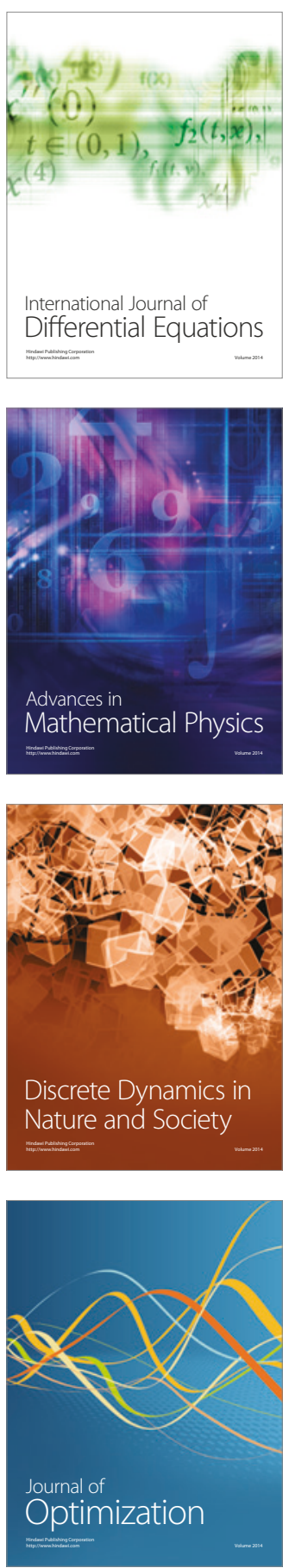